\title{
AN AUGMENTED MIXED-PRIMAL FINITE ELEMENT METHOD FOR A COUPLED FLOW-TRANSPORT PROBLEM*
}

\author{
Mario Alvarez ${ }^{1,2}$, Gabriel N. Gatica ${ }^{2}$ and Ricardo Ruiz-Baier ${ }^{3}$
}

\begin{abstract}
In this paper we analyze the coupling of a scalar nonlinear convection-diffusion problem with the Stokes equations where the viscosity depends on the distribution of the solution to the transport problem. An augmented variational approach for the fluid flow coupled with a primal formulation for the transport model is proposed. The resulting Galerkin scheme yields an augmented mixed-primal finite element method employing Raviart-Thomas spaces of order $k$ for the Cauchy stress, and continuous piecewise polynomials of degree $\leq k+1$ for the velocity and also for the scalar field. The classical Schauder and Brouwer fixed point theorems are utilized to establish existence of solution of the continuous and discrete formulations, respectively. In turn, suitable estimates arising from the connection between a regularity assumption and the Sobolev embedding and Rellich-Kondrachov compactness theorems, are also employed in the continuous analysis. Then, sufficiently small data allow us to prove uniqueness and to derive optimal a priori error estimates. Finally, we report a few numerical tests confirming the predicted rates of convergence, and illustrating the performance of a linearized method based on Newton-Raphson iterations; and we apply the proposed framework in the simulation of thermal convection and sedimentation-consolidation processes.
\end{abstract}

Mathematics Subject Classification. 65N30, 65N12, 76R05, 76D07, 65N15.

Received August 27, 2014. Revised February 16, 2015.

Published online August 19, 2015.

\section{INTRODUCTION}

We are interested in studying mixed finite element approximations to simulate the transport of a species density in an immiscible fluid. Depending on the nature of the species, this problem can be relevant to a number of practical engineering applications including natural and thermal convection, aluminum production, chemical

Keywords and phrases. Stokes equations, nonlinear transport problem, augmented mixed-primal formulation, fixed point theory, thermal convection, sedimentation-consolidation process, finite element methods, a priori error analysis.

* This work was partially supported by CONICYT-Chile through BASAL project CMM, Universidad de Chile, and project Anillo ACT1118 (ANANUM); by the Ministery of Education through the project REDOC.CTA of the Graduate School, Universidad de Concepción; by Centro de Investigación en Ingeniería Matemática (CI $\left.{ }^{2} M A\right)$, Universidad de Concepción; and by the Swiss National Science Foundation through the research grant SNSF PP00P2-144922.

1 Sección de Matemática, Sede Occidente, Universidad de Costa Rica, San Ramón de Alajuela, Costa Rica. mario.alvarez@ucr.ac.cr

$2 \mathrm{CI}^{2} \mathrm{MA}$ and Departamento de Ingeniería Matemática, Universidad de Concepción, Casilla 160-C, Concepción, Chile. mguadamuz@ci2ma.udec.cl; ggatica@ci2ma.udec.cl

3 Institute of Earth Sciences, Géopolis UNIL-Mouline, University of Lausanne, 1015 Lausanne, Switzerland.

ricardo.ruizbaier@unil.ch 
distillation processes, formation of fog, impedance tomography, motion of bio-membranes, semiconductors, granular flows, and so on. In this paper we pay particular attention to the steady state regime in the phenomenon of sedimentation-consolidation of particles (see e.g. $[6,7,30]$ ), where the sought physical quantities in the model include the velocity of the flow and the local solids concentration. On the other hand, it is well known that other variables, such as the principal components of the fluid or solids stress tensors, are of great interest in this context (see e.g. [8], Chap. 3). In a more general sense, the need of obtaining accurate approximations of additional fields has motivated the successful derivation of a wide range of formulations in the framework of Stokes and Navier-Stokes equations, including for instance, stress-velocity formulations (see [9, 16, 20,25] and the references therein). They feature the clear advantage (with respect to classical velocity-pressure formulations) that these auxiliary quantities of interest are computed directly, without resorting to any kind of post-process of the velocity field by numerical differentiation, which usually yields an important loss in accuracy. The attached difficulties are that the finite dimensional spaces involved in the resulting discrete formulation must be properly selected in order to satisfy the corresponding inf-sup condition [5], and that approximation of stresses may become quite expensive if adequate finite elements are not used.

Now, concerning the problem we are interested in here, we realize that, in order to be able to analyze the solvability of a mixed formulation for the fluid flow coupled with a primal method for the transport model, we require $\mathrm{H}^{1}(\Omega)$ smoothness for the components of both the fluid velocity and its discrete approximation. However, since the usual mixed approach is only able to guarantee that they live in $\mathrm{L}^{2}(\Omega)$, in this paper we follow [18] (see also $[17,20]$ ) and propose an augmented mixed scheme in which the stress stays in its original space $\mathbb{H}(\operatorname{div} ; \Omega)$, but the velocity components lie now in the smaller space $\mathrm{H}^{1}(\Omega)$. In other words, the original problem is reformulated as an augmented variational approach for the fluid flow coupled with a primal formulation for the transport model, which constitutes one of the key ideas of this work. According to the above, we will approximate each row of the fluid Cauchy stress tensor with Raviart-Thomas elements of order $k$, whereas the velocity and scalar field (which will represent a solids concentration, or temperature, depending on the specific application) will be approximated with continuous piecewise polynomials of degree $\leq k+1$. The existence of solution of the continuous and associated Galerkin schemes is established by a combination of a suitable regularity assumption, fixed point arguments, the well-know Lax-Milgram theorem, and a classical result on bijective monotone operators. In addition, the Sobolev embedding and Rellich-Kondrachov compactness theorems are also essential in the continuous analysis. Furthermore, the assumption of sufficiently small data allows us to conclude uniqueness of solution and to derive optimal a priori error estimates. The extension of the above described general approach to the more realistic case of steady sedimentation-consolidation systems is under development in [2]. In addition, the incorporation of a similar augmented formulation, and the consequent application of basically the same fixed point theorems employed here, will appear in the forthcoming work [12] where a mixed-primal formulation for the stationary Boussinesq problem is introduced and analyzed.

\section{Outline}

We have organized the contents of this paper as follows. The remainder of this section introduces some standard notation and functional spaces. In Section 2 we first describe the boundary value problem of interest and then slightly simplify it by eliminating the pressure unknown in the fluid. Next, in Section 3, we introduce and analyze the continuous formulation, which is defined by an augmented mixed approach for the fluid flow coupled with a primal method for the transport equation. The necessity of augmentation is clearly justified, and the solvability analysis is based on a fixed point strategy that makes use of the Lax-Milgram and Schauder theorems together with a well-known result on monotone operators. We prove existence of solution and for sufficiently small data we derive uniqueness. The associated Galerkin scheme is introduced in Section 4 by employing Raviart-Thomas elements for the stress, and continuous piecewise polynomial approximations for the velocity and concentration. Here the solvability is established by applying now the Brouwer fixed point theorem and analogous arguments to those employed in Section 3. In Section 5 we assume again sufficiently small data and, applying a suitable Strang-type estimate for nonlinear problems, provide optimal a priori error estimates. Finally, in Section 6 we 
present numerical examples illustrating the good performance of the mixed-primal method and confirming the theoretical rates of convergence.

\section{Preliminaries}

Let us denote by $\Omega \subseteq \mathrm{R}^{n}, n=2,3$ a given bounded domain with polyhedral boundary $\Gamma=\bar{\Gamma}_{\mathrm{D}} \cup \bar{\Gamma}_{\mathrm{N}}$, with $\Gamma_{\mathrm{D}} \cap \Gamma_{\mathrm{N}}=\emptyset$ and $\left|\Gamma_{\mathrm{D}}\right|,\left|\Gamma_{\mathrm{N}}\right|>0$, and denote by $\boldsymbol{\nu}$ the outward unit normal vector on $\Gamma$. Standard notation will be adopted for Lebesgue spaces $\mathrm{L}^{p}(\Omega)$ and Sobolev spaces $\mathrm{H}^{s}(\Omega)$ with norm $\|\cdot\|_{s, \Omega}$ and seminorm $|\cdot|_{s, \Omega}$. In particular, $\mathrm{H}^{1 / 2}(\Gamma)$ is the space of traces of functions of $\mathrm{H}^{1}(\Omega)$ and $\mathrm{H}^{-1 / 2}(\Gamma)$ denotes its dual. By $\mathbf{M}, \mathbb{M}$ we will denote the corresponding vectorial and tensorial counterparts of the generic scalar functional space M. We recall that the space

$$
\mathbb{H}(\operatorname{div} ; \Omega):=\left\{\boldsymbol{\tau} \in \mathbb{L}^{2}(\Omega): \quad \operatorname{div} \boldsymbol{\tau} \in \mathbf{L}^{2}(\Omega)\right\},
$$

equipped with the usual norm

$$
\|\boldsymbol{\tau}\|_{\operatorname{div} ; \Omega}^{2}:=\|\boldsymbol{\tau}\|_{0, \Omega}^{2}+\|\operatorname{div} \boldsymbol{\tau}\|_{0, \Omega}^{2}
$$

is a Hilbert space. As usual, II stands for the identity tensor in $\mathrm{R}^{n \times n}$, and $|\cdot|$ denotes both the Euclidean norm in $\mathrm{R}^{n}$ and the Frobenius norm in $\mathrm{R}^{n \times n}$. Also, for any vector field $\boldsymbol{v}=\left(v_{i}\right)_{i=1, n}$ we set

$$
\nabla \boldsymbol{v}:=\left(\frac{\partial v_{i}}{\partial x_{j}}\right)_{i, j=1, n} \quad \text { and } \quad \operatorname{div} \boldsymbol{v}:=\sum_{j=1}^{n} \frac{\partial v_{j}}{\partial x_{j}} .
$$

In addition, for any tensor fields $\boldsymbol{\tau}=\left(\tau_{i j}\right)_{i, j=1, n}$ and $\boldsymbol{\zeta}=\left(\zeta_{i j}\right)_{i, j=1, n}$, we let $\operatorname{div} \boldsymbol{\tau}$ be the divergence operator div acting along the rows of $\tau$, and define the transpose, the trace, the tensor product, and the deviatoric tensor, respectively, as

$$
\boldsymbol{\tau}^{\mathrm{t}}:=\left(\tau_{j i}\right)_{i, j=1, n}, \quad \operatorname{tr}(\boldsymbol{\tau}):=\sum_{i=1}^{n} \tau_{i i}, \quad \boldsymbol{\tau}: \boldsymbol{\zeta}:=\sum_{i, j=1}^{n} \tau_{i j} \zeta_{i j}, \quad \text { and } \quad \boldsymbol{\tau}^{\mathrm{d}}:=\boldsymbol{\tau}-\frac{1}{n} \operatorname{tr}(\boldsymbol{\tau}) \mathbb{I} .
$$

\section{The MODEL PROBLEM}

The following system of partial differential equations describes the stationary state of the transport of species $\phi$ in an immiscible fluid occupying the domain $\Omega$ :

$$
\begin{gathered}
\boldsymbol{\sigma}=\mu(\phi) \nabla \boldsymbol{u}-p \mathbb{I}, \quad-\operatorname{div} \boldsymbol{\sigma}=\boldsymbol{f} \phi, \quad \operatorname{div} \boldsymbol{u}=0, \\
\tilde{\boldsymbol{\sigma}}=\vartheta(|\nabla \phi|) \nabla \phi-\phi \boldsymbol{u}-\gamma(\phi) \boldsymbol{k}, \quad-\operatorname{div} \tilde{\boldsymbol{\sigma}}=g,
\end{gathered}
$$

where the sought quantities are the Cauchy fluid stress $\boldsymbol{\sigma}$, the local volume-average velocity of the fluid $\boldsymbol{u}$, the pressure $p$, and the local concentration of species $\phi$. For sake of clarity in the presentation, we will restrict ourselves to a specific physical scenario corresponding to the process of sedimentation-consolidation of a mixture (see e.g. [7]). There, it is assumed that a suspension of solid particles within a viscous fluid, undergoes settling due to gravity. The hypotheses of slow sedimentation velocity, short relaxation time, constant density, and negligible expansion viscosities, allow to derive (2.1) from the classical principles of mass and momentum conservation in mixture theory. In this model, the kinematic effective viscosity, $\mu$; the diffusion coefficient, $\vartheta$; and the onedimensional flux function describing hindered settling, $\gamma$; depend nonlinearly on $\phi$, and $\boldsymbol{k}$ is a vector pointing in the direction of gravity. In addition, $\vartheta$ is assumed of class $C^{1}$ and we suppose that there exist positive constants $\mu_{1}, \mu_{2}, \gamma_{1}, \gamma_{2}, \vartheta_{1}$, and $\vartheta_{2}$, such that

$$
\begin{gathered}
\mu_{1} \leq \mu(s) \leq \mu_{2} \quad \text { and } \quad \gamma_{1} \leq \gamma(s) \leq \gamma_{2} \quad \forall s \in \mathrm{R}, \\
\vartheta_{1} \leq \vartheta(s) \leq \vartheta_{2} \quad \text { and } \quad \vartheta_{1} \leq \vartheta(s)+s \vartheta^{\prime}(s) \leq \vartheta_{2} \quad \forall s \geq 0 .
\end{gathered}
$$


Note that (2.2) and the first assumption in (2.3) guarantee, in particular, that the corresponding Nemytsky operators, say $U$ for $\mu$, defined by $U(\phi)(x):=\mu(\phi(x)) \forall \phi \in \mathrm{L}^{2}(\Omega), \forall x \in \Omega$ a.e., and analogously for $\vartheta, \gamma$, $\mu^{-1}, \vartheta^{-1}$, and $\gamma^{-1}$, are all well defined and continuous from $\mathrm{L}^{2}(\Omega)$ into $\mathrm{L}^{2}(\Omega)$. Furthermore, it is easy to show (see, e.g. [23], Thm. 3.8) that the assumptions in (2.3) imply Lipschitz-continuity and strong monotonicity of the nonlinear operator induced by $\vartheta$. We will go back to this fact later on in Section 3.

Some examples of concentration-dependent coefficients typically found in the literature are (see $[6,8,30,31]$ )

$$
\mu(\phi)=\mu_{\infty}\left(1-\frac{\phi}{\phi_{m}}\right)^{-n_{\mu}}, \quad \gamma(\phi)=g_{\infty} \phi(1-\phi)^{n_{\gamma}}, \quad \vartheta(\phi)=\vartheta_{\infty}\left(1-\frac{\phi}{\phi_{m}}\right)^{-n_{\vartheta}},
$$

where $\mu_{\infty}, \phi_{m}, \gamma_{\infty}, n_{\mu}, n_{\gamma}, n_{\vartheta}, \vartheta_{\infty}$ are positive model parameters. These functions violate assumptions (2.2) and (2.3) required in the subsequent analysis. We will therefore consider regularized concentration-dependent coefficients. In turn, some examples of nonlinear functions $\vartheta$ that indeed satisfy $(2.3)$ are the following:

$$
\vartheta(s):=2+\frac{1}{1+s} \quad \text { and } \quad \vartheta(s):=\alpha_{0}+\alpha_{1}\left(1+s^{2}\right)^{(\beta-2) / 2},
$$

where $\alpha_{0}, \alpha_{1}>0$ and $\beta \in[1,2]$. The first example is basically academic but the second one corresponds to a particular case of the well-known Carreau law in fluid mechanics. We also stress that the structure of (2.1) may also serve as prototype model for generalized Boussinesq models and natural convection equations describing the interaction of a fluid driven by gravity and thermal changes. In such a context, $\phi$ can be viewed as the adimensional temperature of the fluid, and typical examples for the variable coefficients (now interpreted as temperature-dependent viscosity and thermal diffusivity) are

$$
\mu(\phi)=\mu_{\infty} \exp (-\phi), \quad \vartheta(\phi)=\vartheta_{\infty} \exp (\phi), \quad \gamma=0,
$$

(see e.g. $[13,15,28])$.

The driving force of the mixture also depends on the local fluctuations of the concentration, so the right hand side of the second equation in (2.1) is linear with respect to $\phi$, and $\boldsymbol{f} \in \mathbf{L}^{\infty}(\Omega)$ and $g \in \mathrm{L}^{2}(\Omega)$ are given functions. Finally, given $\boldsymbol{u}_{\mathrm{D}} \in \mathbf{H}^{1 / 2}\left(\Gamma_{\mathrm{D}}\right)$, the following mixed boundary conditions complement $(2.1)$ :

$$
\boldsymbol{u}=\boldsymbol{u}_{\mathrm{D}} \quad \text { on } \quad \Gamma_{\mathrm{D}}, \quad \boldsymbol{\sigma} \boldsymbol{\nu}=\mathbf{0} \quad \text { on } \quad \Gamma_{\mathrm{N}}, \quad \phi=0 \quad \text { on } \quad \Gamma_{\mathrm{D}}, \quad \text { and } \quad \tilde{\boldsymbol{\sigma}} \cdot \boldsymbol{\nu}=0 \quad \text { on } \quad \Gamma_{\mathrm{N}} .
$$

On the other hand, it is easy to see that the first and third equations in (2.1) are equivalent to

$$
\boldsymbol{\sigma}=\mu(\phi) \nabla \boldsymbol{u}-p \mathbb{I} \quad \text { and } \quad p+\frac{1}{n} \operatorname{tr}(\boldsymbol{\sigma})=0 \quad \text { in } \quad \Omega,
$$

which permits us to eliminate the pressure $p$ from the first equation. Consequently, we arrive at the following coupled system:

$$
\begin{gathered}
\frac{1}{\mu(\phi)} \boldsymbol{\sigma}^{\mathrm{d}}=\nabla \boldsymbol{u} \quad \text { in } \Omega, \quad-\operatorname{div} \boldsymbol{\sigma}=\boldsymbol{f} \phi \text { in } \Omega, \\
\tilde{\boldsymbol{\sigma}}=\vartheta(|\nabla \phi|) \nabla \phi-\phi \boldsymbol{u}-\gamma(\phi) \boldsymbol{k} \quad \text { in } \Omega, \quad-\operatorname{div} \tilde{\boldsymbol{\sigma}}=g \text { in } \Omega, \\
\boldsymbol{u}=\boldsymbol{u}_{\mathrm{D}} \text { on } \Gamma_{\mathrm{D}}, \quad \boldsymbol{\sigma} \boldsymbol{\nu}=\mathbf{0} \quad \text { on } \Gamma_{\mathrm{N}}, \\
\phi=0 \quad \text { on } \Gamma_{\mathrm{D}}, \quad \text { and } \tilde{\boldsymbol{\sigma}} \cdot \boldsymbol{\nu}=0 \text { on } \Gamma_{\mathrm{N}} .
\end{gathered}
$$

We remark here that the incompressibility constraint is implicitly present in the first equation of (2.5), that is in the constitutive equation relating $\boldsymbol{\sigma}$ and $\boldsymbol{u}$. 


\section{The COntinuous Formulation}

\subsection{The augmented mixed-primal formulation}

We first observe that the homogeneous Neumann boundary condition for $\boldsymbol{\sigma}$ on $\Gamma_{N}$ ( $c f$. second relation of (2.4)) suggests to introduce the space

$$
\mathbb{H}_{N}(\operatorname{div} ; \Omega):=\left\{\boldsymbol{\tau} \in \mathbb{H}(\operatorname{div} ; \Omega): \quad \boldsymbol{\tau} \boldsymbol{\nu}=\mathbf{0} \quad \text { on } \quad \Gamma_{\mathrm{N}}\right\} .
$$

Hence, multiplying the first equation of (2.5) by $\boldsymbol{\tau} \in \mathbb{H}_{N}(\operatorname{div} ; \Omega)$, integrating by parts, and using the Dirichlet boundary condition for $\boldsymbol{u}$ ( $c f$. third row of (2.5)), we obtain

$$
\int_{\Omega} \frac{1}{\mu(\phi)} \boldsymbol{\sigma}^{\mathrm{d}}: \boldsymbol{\tau}^{\mathrm{d}}+\int_{\Omega} \boldsymbol{u} \cdot \operatorname{div} \boldsymbol{\tau}=\left\langle\boldsymbol{\tau} \boldsymbol{\nu}, \boldsymbol{u}_{\mathrm{D}}\right\rangle_{\Gamma_{\mathrm{D}}} \quad \forall \boldsymbol{\tau} \in \mathbb{H}_{N}(\operatorname{div} ; \Omega),
$$

where $\langle\cdot, \cdot\rangle_{\Gamma_{\mathrm{D}}}$ is the duality pairing between $\mathbf{H}^{-1 / 2}\left(\Gamma_{\mathrm{D}}\right)$ and $\mathbf{H}^{1 / 2}\left(\Gamma_{\mathrm{D}}\right)$. In addition, the equilibrium equation is then rewritten as

$$
\int_{\Omega} \boldsymbol{v} \cdot \operatorname{div} \boldsymbol{\sigma}=-\int_{\Omega} \boldsymbol{f} \phi \cdot \boldsymbol{v} \quad \forall \boldsymbol{v} \in \mathbf{L}^{2}(\Omega) .
$$

On the other hand, the Dirichlet boundary condition for $\phi$ ( $c f$. fourth row of (2.5)) motivates the introduction of the space

$$
\mathrm{H}_{\Gamma_{\mathrm{D}}}^{1}(\Omega):=\left\{\psi \in \mathrm{H}^{1}(\Omega): \quad \psi=0 \quad \text { on } \quad \Gamma_{\mathrm{D}}\right\},
$$

for which, thanks to the generalized Poincaré inequality, there exists $c_{p}>0$, depending only on $\Omega$ and $\Gamma_{\mathrm{D}}$, such that

$$
\|\psi\|_{1, \Omega} \leq c_{p}|\psi|_{1, \Omega} \quad \forall \psi \in \mathrm{H}_{\Gamma_{\mathrm{D}}}^{1}(\Omega) .
$$

Therefore, given $\phi \in \mathrm{H}_{\Gamma_{\mathrm{D}}}^{1}(\Omega)$, we arrive at the following mixed formulation for the flow: find $(\boldsymbol{\sigma}, \boldsymbol{u}) \in$ $\mathbb{H}_{N}(\operatorname{div} ; \Omega) \times \mathbf{L}^{2}(\Omega)$ such that

$$
\begin{aligned}
a_{\phi}(\boldsymbol{\sigma}, \boldsymbol{\tau})+b(\boldsymbol{\tau}, \boldsymbol{u}) & =\left\langle\boldsymbol{\tau} \boldsymbol{\nu}, \boldsymbol{u}_{\mathrm{D}}\right\rangle_{\Gamma_{\mathrm{D}}} & \forall \boldsymbol{\tau} \in \mathbb{H}_{N}(\operatorname{div} ; \Omega), \\
b(\boldsymbol{\sigma}, \boldsymbol{v}) & =-\int_{\Omega} \boldsymbol{f} \phi \cdot \boldsymbol{v} & \forall \boldsymbol{v} \in \mathbf{L}^{2}(\Omega),
\end{aligned}
$$

where $a_{\phi}: \mathbb{H}_{N}(\operatorname{div} ; \Omega) \times \mathbb{H}_{N}(\operatorname{div} ; \Omega) \rightarrow \mathbb{R}$ and $b: \mathbb{H}_{N}(\operatorname{div} ; \Omega) \times \mathbf{L}^{2}(\Omega) \rightarrow \mathbb{R}$ are bounded bilinear forms defined as

$$
a_{\phi}(\boldsymbol{\zeta}, \boldsymbol{\tau}):=\int_{\Omega} \frac{1}{\mu(\phi)} \boldsymbol{\zeta}^{\mathrm{d}}: \boldsymbol{\tau}^{\mathrm{d}}, \quad b(\boldsymbol{\tau}, \boldsymbol{v}):=\int_{\Omega} \boldsymbol{v} \cdot \operatorname{div} \boldsymbol{\tau},
$$

for $\boldsymbol{\zeta}, \boldsymbol{\tau} \in \mathbb{H}_{N}(\operatorname{div} ; \Omega)$ and $\boldsymbol{v} \in \mathbf{L}^{2}(\Omega)$.

In turn, given $\boldsymbol{u} \in \mathbf{L}^{2}(\Omega)$, and using the homogeneous Neumann boundary condition for $\tilde{\boldsymbol{\sigma}}$ (cf. fourth row of $(2.5)$ ), we deduce that the primal formulation for the concentration equation becomes: find $\phi \in \mathrm{H}_{\Gamma_{\mathrm{D}}}^{1}(\Omega)$ such that

$$
A_{\boldsymbol{u}}(\phi, \psi)=\int_{\Omega} \gamma(\phi) \boldsymbol{k} \cdot \nabla \psi+\int_{\Omega} g \psi \quad \forall \psi \in \mathrm{H}_{\Gamma_{\mathrm{D}}}^{1}(\Omega),
$$

where

$$
A_{\boldsymbol{u}}(\phi, \psi):=\int_{\Omega} \vartheta(|\nabla \phi|) \nabla \phi \cdot \nabla \psi-\int_{\Omega} \phi \boldsymbol{u} \cdot \nabla \psi \quad \forall \phi, \psi \in \mathrm{H}_{\Gamma_{\mathrm{D}}}^{1}(\Omega) .
$$

At this point we observe that the assumption on $\mu$ given in (2.2) and the well known Babuška-Brezzi's theory suffice to show that (3.2) is well-posed (see, e.g. ([24], Thm. 2.1) for details). However, in order to deal with the analysis of (3.3), and particularly to estimate the second term defining $A_{\boldsymbol{u}}$, we would require $\boldsymbol{u} \in \mathbf{H}^{1}(\Omega)$. In fact, 
we now recall from ([28], Eq. (2.20)) that Hölder's inequality and standard Sobolev embeddings estimates ( $c f$. [1], Thm. 4.12, [29], Thm. 1.3.4) yield the existence of a positive constant $c(\Omega)$, depending only on $\Omega$, such that

$$
\left|\int_{\Omega} \varphi \boldsymbol{v} \cdot \nabla \psi\right| \leq c(\Omega)\|\varphi\|_{1, \Omega}\|\boldsymbol{v}\|_{1, \Omega}|\psi|_{1, \Omega} \quad \forall \varphi, \psi \in \mathrm{H}^{1}(\Omega), \quad \forall \boldsymbol{v} \in \mathbf{H}^{1}(\Omega) .
$$

Furthermore, while the exact solution of (3.2) actually satisfies $\nabla \boldsymbol{u}=\frac{1}{\mu(\phi)} \boldsymbol{\sigma}^{\mathrm{d}}$ in $\mathcal{D}^{\prime}(\Omega)$, which implies that $\boldsymbol{u}$ does belong to $\mathbf{H}^{1}(\Omega)$, the foregoing distributional identity does not necessarily extend to the discrete setting of (3.2), and hence the aforementioned difficulty would appear again when trying to analyze the Galerkin scheme associated to (3.3). Therefore, in order to circumvent this inconvenience, we proceed similarly as in ([18], Sect. 3) and incorporate into (3.2) the following redundant Galerkin terms

$$
\begin{array}{rlrl}
\kappa_{1} \int_{\Omega}\left(\nabla \boldsymbol{u}-\frac{1}{\mu(\phi)} \boldsymbol{\sigma}^{\mathrm{d}}\right): \nabla \boldsymbol{v} & =0 & & \forall \boldsymbol{v} \in \mathbf{H}^{1}(\Omega), \\
\kappa_{2} \int_{\Omega} \operatorname{div} \boldsymbol{\sigma} \cdot \operatorname{div} \boldsymbol{\tau}=-\kappa_{2} \int_{\Omega} \boldsymbol{f} \phi \cdot \operatorname{div} \boldsymbol{\tau} & & \forall \boldsymbol{\tau} \in \mathbb{H}_{N}(\operatorname{div} ; \Omega), \\
\kappa_{3} \int_{\Gamma_{\mathrm{D}}} \boldsymbol{u} \cdot \boldsymbol{v} & =\kappa_{3} \int_{\Gamma_{\mathrm{D}}}^{\boldsymbol{u}_{\mathrm{D}} \cdot \boldsymbol{v}} & & \forall \boldsymbol{v} \in \mathbf{H}^{1}(\Omega),
\end{array}
$$

where $\left(\kappa_{1}, \kappa_{2}, \kappa_{3}\right)$ is a vector of positive parameters to be specified later. Notice that the first and third equations in (3.6) implicitly require the velocity $\boldsymbol{u}$ to live in $\mathbf{H}^{1}(\Omega)$. In this way, instead of (3.2), we consider from now on the following augmented mixed formulation: find $(\boldsymbol{\sigma}, \boldsymbol{u}) \in \mathbb{H}_{N}(\operatorname{div} ; \Omega) \times \mathbf{H}^{1}(\Omega)$ such that

$$
B_{\phi}((\boldsymbol{\sigma}, \boldsymbol{u}),(\boldsymbol{\tau}, \boldsymbol{v}))=F_{\phi}(\boldsymbol{\tau}, \boldsymbol{v}) \quad \forall(\boldsymbol{\tau}, \boldsymbol{v}) \in \mathbb{H}_{N}(\operatorname{div} ; \Omega) \times \mathbf{H}^{1}(\Omega),
$$

where

$$
\begin{aligned}
B_{\phi}((\boldsymbol{\sigma}, \boldsymbol{u}),(\boldsymbol{\tau}, \boldsymbol{v})):= & a_{\phi}(\boldsymbol{\sigma}, \boldsymbol{\tau})+b(\boldsymbol{\tau}, \boldsymbol{u})-b(\boldsymbol{\sigma}, \boldsymbol{v})+\kappa_{1} \int_{\Omega}\left(\nabla \boldsymbol{u}-\frac{1}{\mu(\phi)} \boldsymbol{\sigma}^{\mathrm{d}}\right): \nabla \boldsymbol{v} \\
& +\kappa_{2} \int_{\Omega} \operatorname{div} \boldsymbol{\sigma} \cdot \operatorname{div} \boldsymbol{\tau}+\kappa_{3} \int_{\Gamma_{\mathrm{D}}} \boldsymbol{u} \cdot \boldsymbol{v}
\end{aligned}
$$

and

$$
F_{\phi}(\boldsymbol{\tau}, \boldsymbol{v}):=\left\langle\boldsymbol{\tau} \boldsymbol{\nu}, \boldsymbol{u}_{\mathrm{D}}\right\rangle_{\Gamma_{\mathrm{D}}}+\int_{\Omega} \boldsymbol{f} \phi \cdot \boldsymbol{v}-\kappa_{2} \int_{\Omega} \boldsymbol{f} \phi \cdot \operatorname{div} \boldsymbol{\tau}+\kappa_{3} \int_{\Gamma_{\mathrm{D}}} \boldsymbol{u}_{\mathrm{D}} \cdot \boldsymbol{v} .
$$

We remark in advance that the well-posedness of (3.7) is proved below in Section 3.3. In particular, we emphasize that the positiveness of the parameter $\kappa_{2}$ in (3.8) is crucial for the ellipticity of the bilinear form $B_{\phi}$ in the product space $\mathbb{H}_{N}(\operatorname{div} ; \Omega) \times \mathbf{H}^{1}(\Omega)$ (see below (3.18) and (3.19) in the proof of Lem. 3.4), which enables to choose arbitrary finite element subspaces to define the associated discrete formulation. Otherwise, one would need inf-sup conditions for the bilinear form $b$, which, involving the spaces $\mathbb{H}_{N}(\mathbf{d i v} ; \Omega)$ and $\mathbf{H}^{1}(\Omega)$, and suitable subspaces of them, do not seem to be easily verifiable. In turn, the term multiplying $\kappa_{2}$ in (3.8) just adds a minor complexity to the Galerkin scheme since the corresponding matrix becomes block-diagonal. On the other hand, since the unique solution of (3.2) is obviously a solution of (3.7) as well, we will conclude that both continuous problems share the same unique solution.

In this way, the augmented mixed-primal formulation of our original coupled problem (2.5) reduces to (3.7) and (3.3), that is: find $(\boldsymbol{\sigma}, \boldsymbol{u}, \phi) \in \mathbb{H}_{N}(\operatorname{div} ; \Omega) \times \mathbf{H}^{1}(\Omega) \times \mathrm{H}_{\Gamma_{\mathrm{D}}}^{1}(\Omega)$ such that

$$
\begin{aligned}
B_{\phi}((\boldsymbol{\sigma}, \boldsymbol{u}),(\boldsymbol{\tau}, \boldsymbol{v})) & =F_{\phi}(\boldsymbol{\tau}, \boldsymbol{v}) \quad \forall(\boldsymbol{\tau}, \boldsymbol{v}) \in \mathbb{H}_{N}(\operatorname{div} ; \Omega) \times \mathbf{H}^{1}(\Omega), \\
A_{\boldsymbol{u}}(\phi, \psi) & =\int_{\Omega} \gamma(\phi) \boldsymbol{k} \cdot \nabla \psi+\int_{\Omega} g \psi \quad \forall \psi \in \mathrm{H}_{\Gamma_{\mathrm{D}}}^{1}(\Omega) .
\end{aligned}
$$




\subsection{A fixed point strategy}

Having proposed the alternative formulation (3.7), whose continuous and discrete solutions have second components living in $\mathbf{H}^{1}(\Omega)$, we are able now to take a second look at (3.3). More precisely, given $\phi \in \mathrm{H}_{\Gamma_{\mathrm{D}}}^{1}(\Omega)$ and the corresponding solution $(\boldsymbol{\sigma}, \boldsymbol{u}) \in \mathbb{H}_{N}(\operatorname{div} ; \Omega) \times \mathbf{H}^{1}(\Omega)$ of (3.7), we can set, instead of (3.3), the modified primal formulation: find $\widetilde{\phi} \in \mathrm{H}_{\Gamma_{\mathrm{D}}}^{1}(\Omega)$ such that

$$
A_{\boldsymbol{u}}(\widetilde{\phi}, \widetilde{\psi})=G_{\phi}(\widetilde{\psi}) \quad \forall \widetilde{\psi} \in \mathrm{H}_{\Gamma_{\mathrm{D}}}^{1}(\Omega),
$$

where

$$
G_{\phi}(\widetilde{\psi}):=\int_{\Omega} \gamma(\phi) \boldsymbol{k} \cdot \nabla \widetilde{\psi}+\int_{\Omega} g \widetilde{\psi} \quad \forall \widetilde{\psi} \in \mathrm{H}_{\Gamma_{\mathrm{D}}}^{1}(\Omega) .
$$

The well-posedness of this nonlinear problem will also be addressed in Section 3.3. Alternatively, one could also deal, instead of (3.11), with the linear problem: find $\widetilde{\phi} \in \mathrm{H}_{\Gamma_{\mathrm{D}}}^{1}(\Omega)$ such that

$$
A_{\phi, \boldsymbol{u}}(\widetilde{\phi}, \widetilde{\psi})=G_{\phi}(\widetilde{\psi}) \quad \forall \widetilde{\psi} \in \mathrm{H}_{\Gamma_{\mathrm{D}}}^{1}(\Omega),
$$

where

$$
A_{\phi, \boldsymbol{u}}(\widetilde{\phi}, \widetilde{\psi}):=\int_{\Omega} \vartheta(|\nabla \phi|) \nabla \widetilde{\phi} \cdot \nabla \widetilde{\psi}-\int_{\Omega} \widetilde{\phi} \boldsymbol{u} \cdot \nabla \widetilde{\psi} \quad \forall \widetilde{\phi}, \widetilde{\psi} \in \mathrm{H}_{\Gamma_{\mathrm{D}}}^{1}(\Omega) .
$$

Nevertheless, and for easiness of the analysis, throughout the rest of the paper we stay with (3.11).

Hence, the description of problems (3.7) and (3.11) suggests a fixed point strategy to analyze (3.10). Indeed, let $\mathbf{S}: \mathrm{H}_{\Gamma_{\mathrm{D}}}^{1}(\Omega) \longrightarrow \mathbb{H}_{N}(\operatorname{div} ; \Omega) \times \mathbf{H}^{1}(\Omega)$ be the operator defined by:

$$
\mathbf{S}(\phi)=\left(\mathbf{S}_{1}(\phi), \mathbf{S}_{2}(\phi)\right):=(\boldsymbol{\sigma}, \boldsymbol{u}) \in \mathbb{H}_{N}(\operatorname{div} ; \Omega) \times \mathbf{H}^{1}(\Omega) \quad \forall \phi \in \mathrm{H}_{\Gamma_{\mathrm{D}}}^{1}(\Omega),
$$

where $(\boldsymbol{\sigma}, \boldsymbol{u})$ is the unique solution of $(3.7)$ with the given $\phi$. In turn, let $\widetilde{\mathbf{S}}: \mathrm{H}_{\Gamma_{\mathrm{D}}}^{1}(\Omega) \times \mathbf{H}^{1}(\Omega) \longrightarrow \mathrm{H}_{\Gamma_{\mathrm{D}}}^{1}(\Omega)$ be the operator defined by

$$
\widetilde{\mathbf{S}}(\phi, \boldsymbol{u}):=\widetilde{\phi} \quad \forall(\phi, \boldsymbol{u}) \in \mathrm{H}_{\Gamma_{\mathrm{D}}}^{1}(\Omega) \times \mathbf{H}^{1}(\Omega),
$$

where $\widetilde{\phi}$ is the unique solution of $(3.11)$ with the given $(\phi, \boldsymbol{u})$. Then, we define the operator $\mathbf{T}: \mathrm{H}_{\Gamma_{\mathrm{D}}}^{1}(\Omega) \longrightarrow$ $\mathrm{H}_{\Gamma_{\mathrm{D}}}^{1}(\Omega)$ by

$$
\mathbf{T}(\phi):=\widetilde{\mathbf{S}}\left(\phi, \mathbf{S}_{2}(\phi)\right) \quad \forall \phi \in \mathrm{H}_{\Gamma_{\mathrm{D}}}^{1}(\Omega),
$$

and realize that solving (3.10) is equivalent to seeking a fixed point of $\mathbf{T}$, that is: find $\phi \in \mathrm{H}_{\Gamma_{\mathrm{D}}}^{1}(\Omega)$ such that

$$
\mathbf{T}(\phi)=\phi .
$$

\subsection{Well-posedness of the uncoupled problems}

In this section we show that the uncoupled problems (3.7) and (3.11) are in fact well-posed. We begin by recalling (see, e.g. [5]) that $\mathbb{H}(\operatorname{div} ; \Omega)=\mathbb{H}_{0}(\operatorname{div} ; \Omega) \oplus \mathrm{R} \mathbb{I}$, where

$$
\mathbb{H}_{0}(\operatorname{div} ; \Omega):=\left\{\zeta \in \mathbb{H}(\operatorname{div} ; \Omega): \quad \int_{\Omega} \operatorname{tr}(\boldsymbol{\zeta})=0\right\}
$$

More precisely, for each $\boldsymbol{\zeta} \in \mathbb{H}(\mathbf{d i v} ; \Omega)$ there exist unique $\boldsymbol{\zeta}_{0}:=\boldsymbol{\zeta}-\left\{\frac{1}{n|\Omega|} \int_{\Omega} \operatorname{tr}(\boldsymbol{\zeta})\right\} \mathbb{I} \in \mathbb{H}_{0}(\operatorname{div} ; \Omega)$ and $d:=\frac{1}{n|\Omega|} \int_{\Omega} \operatorname{tr}(\boldsymbol{\zeta}) \in \mathbb{R}$, such that $\boldsymbol{\zeta}=\boldsymbol{\zeta}_{0}+d \mathbb{I}$. The following three lemmas from $[5,18,20]$, which concern the above decomposition and an equivalence of norm, will be employed to show the well-posedness of (3.7) for a given $\phi$. 
Lemma 3.1. There exists $c_{1}=c_{1}(\Omega)>0$ such that

$$
c_{1}\left\|\boldsymbol{\tau}_{0}\right\|_{0, \Omega}^{2} \leq\left\|\boldsymbol{\tau}^{\mathrm{d}}\right\|_{0, \Omega}^{2}+\|\operatorname{div} \boldsymbol{\tau}\|_{0, \Omega}^{2} \quad \forall \boldsymbol{\tau}=\boldsymbol{\tau}_{0}+c \mathbb{I} \in \mathbb{H}(\operatorname{div} ; \Omega),
$$

with $\boldsymbol{\tau}_{0} \in \mathbb{H}_{0}(\operatorname{div} ; \Omega)$ and $c \in \mathrm{R}$.

Proof. (see [5], Prop. 3.1).

Lemma 3.2. There exists $c_{2}=c_{2}\left(\Omega, \Gamma_{\mathrm{N}}\right)>0$ such that

$$
c_{2}\|\boldsymbol{\tau}\|_{\text {div } ; \Omega}^{2} \leq\left\|\boldsymbol{\tau}_{0}\right\|_{\operatorname{div} ; \Omega}^{2} \quad \forall \boldsymbol{\tau}=\boldsymbol{\tau}_{0}+c \mathbb{I} \in \mathbb{H}_{N}(\operatorname{div} ; \Omega),
$$

with $\boldsymbol{\tau}_{0} \in \mathbb{H}_{0}(\operatorname{div} ; \Omega)$ and $c \in \mathrm{R}$.

Proof. (see [20], Lem. 2.2).

Lemma 3.3. There exists $c_{3}=c_{3}\left(\Omega, \Gamma_{\mathrm{D}}\right)>0$ such that

$$
|\boldsymbol{v}|_{1, \Omega}^{2}+\|\boldsymbol{v}\|_{0, \Gamma_{\mathrm{D}}}^{2} \geq c_{3}\|\boldsymbol{v}\|_{1, \Omega}^{2} \quad \forall \boldsymbol{v} \in \mathbf{H}^{1}(\Omega) .
$$

Proof. It corresponds to a slight modification of the proof of ([18], Lem. 3.3).

Furthermore, for sake of the subsequent analysis we will also require Lipschitz continuity assumptions for $\gamma$ and $\mu$. More precisely, we assume that there exist positive constants $L_{\gamma}$ and $L_{\mu}$ such that

$$
|\gamma(s)-\gamma(t)| \leq L_{\gamma}|s-t| \quad \forall s, t \in \mathrm{R},
$$

and

$$
|\mu(s)-\mu(t)| \leq L_{\mu}|s-t| \quad \forall s, t \in \mathrm{R} .
$$

We now begin the solvability analysis of the uncoupled problems with the following result.

Lemma 3.4. Assume that $\kappa_{1} \in\left(0, \frac{2 \delta \mu_{1}}{\mu_{2}}\right)$ with $\delta \in\left(0,2 \mu_{1}\right)$, and that $0<\kappa_{2}, \kappa_{3}$. Then, for each $\phi \in \mathrm{H}_{\Gamma_{\mathrm{D}}}^{1}(\Omega)$ the problem (3.7) has a unique solution $\mathbf{S}(\phi):=(\boldsymbol{\sigma}, \boldsymbol{u}) \in H:=\mathbb{H}_{N}(\mathbf{d i v} ; \Omega) \times \mathbf{H}^{1}(\Omega)$. Moreover, there exists $C_{S}>0$, independent of $\phi$, such that

$$
\|\mathbf{S}(\phi)\|_{H}=\|(\boldsymbol{\sigma}, \boldsymbol{u})\|_{H} \leq C_{S}\left\{\left\|\boldsymbol{u}_{\mathrm{D}}\right\|_{1 / 2, \Gamma_{\mathrm{D}}}+\|\boldsymbol{f}\|_{\infty, \Omega}\|\phi\|_{0, \Omega}\right\} \quad \forall \phi \in \mathrm{H}_{\Gamma_{\mathrm{D}}}^{1}(\Omega) .
$$

Proof. We first observe from (3.8) that, given $\phi \in \mathrm{H}_{\Gamma_{\mathrm{D}}}^{1}(\Omega), B_{\phi}$ is clearly a bilinear form. Next, applying the Cauchy-Schwarz's inequality, the lower bound for $\mu(c f .(2.2))$, and the trace theorem (with constant $c_{0}$ ), we also obtain from (3.8) that

$$
\begin{gathered}
\quad\left|B_{\phi}((\boldsymbol{\sigma}, \boldsymbol{u}),(\boldsymbol{\tau}, \boldsymbol{v}))\right| \leq \frac{1}{\mu_{1}}\left\|\boldsymbol{\sigma}^{\mathrm{d}}\right\|_{0, \Omega}\left\|\boldsymbol{\tau}^{\mathrm{d}}\right\|_{0, \Omega}+\|\boldsymbol{u}\|_{0, \Omega}\|\operatorname{div} \boldsymbol{\tau}\|_{0, \Omega}+\|\boldsymbol{v}\|_{0, \Omega}\|\operatorname{div} \boldsymbol{\sigma}\|_{0, \Omega} \\
+\kappa_{1}|\boldsymbol{u}|_{1, \Omega}|\boldsymbol{v}|_{1, \Omega}+\frac{\kappa_{1}}{\mu_{1}}\left\|\boldsymbol{\sigma}^{\mathrm{d}}\right\|_{0, \Omega}|\boldsymbol{v}|_{1, \Omega}+\kappa_{2}\|\operatorname{div} \boldsymbol{\sigma}\|_{0, \Omega}\|\operatorname{div} \boldsymbol{\tau}\|_{0, \Omega}+c_{0}^{2} \kappa_{3}\|\boldsymbol{u}\|_{1, \Omega}\|\boldsymbol{v}\|_{1, \Omega}
\end{gathered}
$$

It follows that there exists a positive constant, denoted $\|B\|$ and depending on $\mu_{1}, \kappa_{1}, \kappa_{2}, \kappa_{3}$, and $c_{0}$, such that

$$
\left|B_{\phi}((\boldsymbol{\sigma}, \boldsymbol{u}),(\boldsymbol{\tau}, \boldsymbol{v}))\right| \leq\|B\|\|(\boldsymbol{\sigma}, \boldsymbol{u})\|_{H}\|(\boldsymbol{\tau}, \boldsymbol{v})\|_{H} \quad \forall(\boldsymbol{\sigma}, \boldsymbol{u}),(\boldsymbol{\tau}, \boldsymbol{v}) \in H,
$$

and hence $B_{\phi}$ is bounded independently of $\phi \in \mathrm{H}_{\Gamma_{\mathrm{D}}}^{1}(\Omega)$.

In turn, we now aim to show that $B_{\phi}$ is $H$-elliptic. In fact, given $(\boldsymbol{\tau}, \boldsymbol{v}) \in H$, we have again from (3.8) that

$$
B_{\phi}((\boldsymbol{\tau}, \boldsymbol{v}),(\boldsymbol{\tau}, \boldsymbol{v}))=\int_{\Omega} \frac{1}{\mu(\phi)} \boldsymbol{\tau}^{\mathrm{d}}: \boldsymbol{\tau}^{\mathrm{d}}+\kappa_{1}|\boldsymbol{v}|_{1, \Omega}^{2}-\kappa_{1} \int_{\Omega} \frac{1}{\mu(\phi)} \boldsymbol{\tau}^{\mathrm{d}}: \nabla \boldsymbol{v}+\kappa_{2}\|\operatorname{div} \boldsymbol{\tau}\|_{0, \Omega}^{2}+\kappa_{3}\|\boldsymbol{v}\|_{0, \Gamma_{\mathrm{D}}}^{2},
$$


which, using the bounds for $\mu$ (cf. (2.2)), the Young inequality, and Lemmas 3.1, 3.2, and 3.3, and taking $\delta, \kappa_{1}$, $\kappa_{2}$, and $\kappa_{3}$ as stated in the hypotheses, yields

$$
\begin{aligned}
B_{\phi}((\boldsymbol{\tau}, \boldsymbol{v}) & ,(\boldsymbol{\tau}, \boldsymbol{v})) \geq\left(\frac{1}{\mu_{2}}-\frac{\kappa_{1}}{2 \delta \mu_{1}}\right)\left\|\boldsymbol{\tau}^{\mathrm{d}}\right\|_{0, \Omega}^{2}+\kappa_{2}\|\operatorname{div} \boldsymbol{\tau}\|_{0, \Omega}^{2}+\kappa_{1}\left(1-\frac{\delta}{2 \mu_{1}}\right)|\boldsymbol{v}|_{1, \Omega}^{2}+\kappa_{3}\|\boldsymbol{v}\|_{0, \Gamma_{\mathrm{D}}}^{2} \\
& \geq c_{1} \alpha_{1}\left\|\boldsymbol{\tau}_{0}\right\|_{0, \Omega}^{2}+\frac{\kappa_{2}}{2}\|\operatorname{div} \boldsymbol{\tau}\|_{0, \Omega}^{2}+\kappa_{1}\left(1-\frac{\delta}{2 \mu_{1}}\right)|\boldsymbol{v}|_{1, \Omega}^{2}+\kappa_{3}\|\boldsymbol{v}\|_{0, \Gamma_{\mathrm{D}}}^{2} \\
& \geq \alpha_{2}\left\|\boldsymbol{\tau}_{0}\right\|_{\operatorname{div} ; \Omega}^{2}+\alpha_{3}\left\{|\boldsymbol{v}|_{1, \Omega}^{2}+\|\boldsymbol{v}\|_{0, \Gamma_{\mathrm{D}}}^{2}\right\} \\
& \geq c_{2} \alpha_{2}\|\boldsymbol{\tau}\|_{\operatorname{div} ; \Omega}^{2}+c_{3} \alpha_{3}\|\boldsymbol{v}\|_{1, \Omega}^{2},
\end{aligned}
$$

where $\alpha_{1}:=\min \left\{\left(\frac{1}{\mu_{2}}-\frac{\kappa_{1}}{2 \delta \mu_{1}}\right), \frac{\kappa_{2}}{2}\right\}, \alpha_{2}:=\min \left\{c_{1} \alpha_{1}, \frac{\kappa_{2}}{2}\right\}$, and $\alpha_{3}:=\min \left\{\kappa_{1}\left(1-\frac{\delta}{2 \mu_{1}}\right), \kappa_{3}\right\}$. In this way, defining $\alpha:=\min \left\{c_{2} \alpha_{2}, c_{3} \alpha_{3}\right\}$, which depends on $\mu_{1}, \mu_{2}, \delta, \kappa_{1}, \kappa_{2}, \kappa_{3}, c_{1}, c_{2}$, and $c_{3}$, we conclude that

$$
B_{\phi}((\boldsymbol{\tau}, \boldsymbol{v}),(\boldsymbol{\tau}, \boldsymbol{v})) \geq \alpha\|(\boldsymbol{\tau}, \boldsymbol{v})\|_{H}^{2} \quad \forall(\boldsymbol{\tau}, \boldsymbol{v}) \in H,
$$

thus confirming the $H$-ellipticity of $B_{\phi}$ independently of $\phi \in \mathrm{H}_{\Gamma_{\mathrm{D}}}^{1}(\Omega)$ as well. In particular, choosing the feasible values $\delta=\mu_{1}$ and $\kappa_{1}=\frac{\mu_{1}^{2}}{\mu_{2}}$, and then taking $\kappa_{2}=2\left(\frac{1}{\mu_{2}}-\frac{\kappa_{1}}{2 \delta \mu_{1}}\right)$ and $\kappa_{3}=\kappa_{1}\left(1-\frac{\delta}{2 \mu_{1}}\right)$, we find that $\kappa_{2}=\frac{1}{\mu_{2}}$, $\kappa_{3}=\frac{\mu_{1}^{2}}{2 \mu_{2}}$, and $\alpha=\frac{1}{2 \mu_{2}} \min \left\{c_{1} c_{2}, c_{2}, c_{3} \mu_{1}^{2}\right\}$.

Next, given $\phi \in \mathrm{H}_{\Gamma_{\mathrm{D}}}^{1}(\Omega)$, we look at the functional $F_{\phi}$ (cf. (3.9)), which is certainly linear. Then, using the Cauchy-Schwarz's inequality and the trace estimates in $\mathbb{H}(\operatorname{div} ; \Omega)$ and $\mathbf{H}^{1}(\Omega)$, with constants 1 and $c_{0}$, respectively, we deduce that for each $(\boldsymbol{\tau}, \boldsymbol{v}) \in H$ there holds

$$
\begin{gathered}
\left|F_{\phi}(\boldsymbol{\tau}, \boldsymbol{v})\right| \leq\|\boldsymbol{\tau}\|_{\operatorname{div} ; \Omega}\left\|\boldsymbol{u}_{\mathrm{D}}\right\|_{1 / 2, \Gamma_{\mathrm{D}}}+\|\boldsymbol{f}\|_{\infty, \Omega}\|\phi\|_{0, \Omega}\left\{\|\boldsymbol{v}\|_{0, \Omega}+\kappa_{2}\|\operatorname{div} \boldsymbol{\tau}\|_{0, \Omega}\right\} \\
+c_{0} \kappa_{3}\left\|\boldsymbol{u}_{\mathrm{D}}\right\|_{1 / 2, \Gamma_{\mathrm{D}}}\|\boldsymbol{v}\|_{1, \Omega},
\end{gathered}
$$

which provides the existence of a positive constant, denoted $\|F\|$ and depending on $\kappa_{2}, \kappa_{3}$, and $c_{0}$, such that

$$
\left|F_{\phi}(\boldsymbol{\tau}, \boldsymbol{v})\right| \leq\|F\|\left\{\left\|\boldsymbol{u}_{\mathrm{D}}\right\|_{1 / 2, \Gamma_{\mathrm{D}}}+\|\boldsymbol{f}\|_{\infty, \Omega}\|\phi\|_{0, \Omega}\right\}\|(\boldsymbol{\tau}, \boldsymbol{v})\| \quad \forall(\boldsymbol{\tau}, \boldsymbol{v}) \in H .
$$

The foregoing inequality shows the boundedness of $F_{\phi}$ with

$$
\left\|F_{\phi}\right\| \leq\|F\|\left\{\left\|\boldsymbol{u}_{\mathrm{D}}\right\|_{1 / 2, \Gamma_{\mathrm{D}}}+\|\boldsymbol{f}\|_{\infty, \Omega}\|\phi\|_{0, \Omega}\right\} .
$$

Finally, a straightforward application of the Lax-Milgram's Lemma (see, e.g. [21], Thm. 1.1), proves that, for each $\phi \in \mathrm{H}_{\Gamma_{\mathrm{D}}}^{1}(\Omega)$, problem (3.7) has a unique solution $\mathbf{S}(\phi):=(\boldsymbol{\sigma}, \boldsymbol{u}) \in H$. Moreover, the corresponding continuous dependence result together with the estimates (3.19) and (3.20) give

$$
\|\mathbf{S}(\phi)\|_{H}=\|(\boldsymbol{\sigma}, \boldsymbol{u})\|_{H} \leq \frac{1}{\alpha}\left\|F_{\phi}\right\|_{H^{\prime}} \leq C_{S}\left\{\left\|\boldsymbol{u}_{\mathrm{D}}\right\|_{1 / 2, \Gamma_{\mathrm{D}}}+\|\boldsymbol{f}\|_{\infty, \Omega}\|\phi\|_{0, \Omega}\right\},
$$

with $C_{S}:=\frac{\|F\|}{\alpha}$, thus completing the proof.

Throughout the rest of the paper we suppose further regularity for the problem defining the operator $\mathbf{S}$. More precisely, we assume that $\boldsymbol{u}_{\mathrm{D}} \in \mathbf{H}^{1 / 2+\varepsilon}\left(\Gamma_{\mathrm{D}}\right)$ for some $\varepsilon \in(0,1)$ (when $n=2$ ) or $\varepsilon \in\left(\frac{1}{2}, 1\right)$ (when $n=3$ ), and that for each $\psi \in \mathrm{H}_{\Gamma_{\mathrm{D}}}^{1}(\Omega)$ with $\|\psi\|_{1, \Omega} \leq r, r>0$ given, there hold $(\boldsymbol{\zeta}, \boldsymbol{w}):=\mathbf{S}(\psi) \in \mathbb{H}_{N}(\operatorname{div} ; \Omega) \cap \mathbb{H}^{\varepsilon}(\Omega) \times$ $\mathbf{H}^{1+\varepsilon}(\Omega)$ and

$$
\|\boldsymbol{\zeta}\|_{\varepsilon, \Omega}+\|\boldsymbol{w}\|_{1+\varepsilon, \Omega} \leq \widetilde{C}_{S}(r)\left\{\left\|\boldsymbol{u}_{\mathrm{D}}\right\|_{1 / 2+\varepsilon, \Gamma_{\mathrm{D}}}+\|\boldsymbol{f}\|_{\infty, \Omega}\|\psi\|_{0, \Omega}\right\}
$$


with a positive constant $\widetilde{C}_{S}(r)$ independent of the given $\psi$ but depending on the upper bound $r$ of its $\mathrm{H}^{1}$-norm. We remark that the reason of the stipulated ranges for $\varepsilon$ will be clarified in the forthcoming analysis (see below proof of Lem. 3.12). In turn, while the actual verification of (3.22) is beyond the goals of the present work, we observe that the fact that (3.2) and (3.7) share the same solution implies that this issue reduces finally to the regularity of the Stokes problem with variable viscosity $\mu$ depending on $\phi$ (see, e.g. [4] for analogous regularity results). To this respect, we would like to mention that the equilibrium equation $-\operatorname{div} \boldsymbol{\zeta}=\boldsymbol{f} \psi$ in $\Omega$, obviously controls $\operatorname{div} \boldsymbol{\zeta}$, whereas the constitutive equation $\frac{1}{\mu(\psi)} \boldsymbol{\zeta}^{\mathrm{d}}=\nabla \boldsymbol{w}$ in $\Omega$, may serve to control the curl of $\boldsymbol{\zeta}$. Certainly, the Lipschitz-continuity of $\mu$ (cf. (3.15)) and the upper bound of $\|\psi\|_{1, \Omega}$ are essential here. In addition, the Dirichlet boundary condition on $\boldsymbol{w}$ should be used under the form of its tangential derivative, and the eventual presence of corners in $\Gamma$ should not be a problem.

According to the above, the present assumption is indeed quite reasonable at least for $n=2$ since just $\varepsilon \in(0,1)$ is required in this case. However, due to the hypothesis $\varepsilon \in\left(\frac{1}{2}, 1\right)$ when $n=3$, we conjecture that mixed boundary conditions for $\boldsymbol{u}$ and $\boldsymbol{\sigma}$ will have to be excluded of the corresponding 3D problem and that either Dirichlet or Neumann boundary conditions only will be allowed. Finally, and while the estimate (3.22) will be employed only to bound $\|\boldsymbol{\zeta}\|_{\varepsilon, \Omega}$, we have stated it including the term $\|\boldsymbol{w}\|_{1+\varepsilon, \Omega}$ since, because of the constitutive equation, the regularities of $\boldsymbol{\zeta}$ and $\boldsymbol{w}$ will most likely be connected.

We now establish the unique solvability of the nonlinear problem (3.11).

Lemma 3.5. Let $\phi \in \mathrm{H}_{\Gamma_{\mathrm{D}}}^{1}(\Omega)$ and $\boldsymbol{u} \in \mathbf{H}^{1}(\Omega)$ such that $\|\boldsymbol{u}\|_{1, \Omega}<\frac{\vartheta_{1}}{c_{p} c(\Omega)}$ (cf. (2.3), (3.1), (3.5)). Then, there exists a unique $\widetilde{\phi}:=\widetilde{\mathbf{S}}(\phi, \boldsymbol{u}) \in \mathrm{H}_{\Gamma_{\mathrm{D}}}^{1}(\Omega)$ solution of (3.11), and there holds

$$
\|\widetilde{\mathbf{S}}(\phi, \boldsymbol{u})\|_{1, \Omega}=\|\widetilde{\phi}\|_{1, \Omega} \leq \frac{c_{p}^{2}}{\left(\vartheta_{1}-c_{p} c(\Omega)\|\boldsymbol{u}\|_{1, \Omega}\right)}\left\{\gamma_{2}|\Omega|^{1 / 2}\|\boldsymbol{k}\|+\|g\|_{0, \Omega}\right\} .
$$

Proof. We begin by recalling from ([23], Thm. 3.8) that the nonlinear operator induced by the first term defining $A_{\boldsymbol{u}}$ (cf. (3.4)) is strongly monotone and Lipschitz-continuous with constants $\vartheta_{1}$ and $\widetilde{\vartheta}_{2}:=\max \left\{\vartheta_{2}, 2 \vartheta_{2}-\vartheta_{1}\right\}$ (cf. (2.3)), respectively. It follows, using also the Cauchy-Schwarz's inequality, (3.5), and (3.1), that for all $\widetilde{\varphi}, \widetilde{\psi} \in \mathrm{H}_{\Gamma_{\mathrm{D}}}^{1}(\Omega)$ there holds

$$
\begin{aligned}
A_{\boldsymbol{u}}(\widetilde{\varphi}, \widetilde{\varphi} & -\widetilde{\psi})-A_{\boldsymbol{u}}(\widetilde{\psi}, \widetilde{\varphi}-\widetilde{\psi}) \\
& =\int_{\Omega}\{\vartheta(|\nabla \widetilde{\varphi}|) \nabla \widetilde{\varphi}-\vartheta(|\nabla \widetilde{\psi}|) \nabla \widetilde{\psi}\} \cdot \nabla(\widetilde{\varphi}-\widetilde{\psi})-\int_{\Omega}(\widetilde{\varphi}-\widetilde{\psi}) \boldsymbol{u} \cdot \nabla(\widetilde{\varphi}-\widetilde{\psi}) \\
& \geq \vartheta_{1}|\widetilde{\varphi}-\widetilde{\psi}|_{1, \Omega}^{2}-c(\Omega)\|\widetilde{\varphi}-\widetilde{\psi}\|_{1, \Omega}\|\boldsymbol{u}\|_{1, \Omega}|\widetilde{\varphi}-\widetilde{\psi}|_{1, \Omega} \\
& \geq\left\{\vartheta_{1}-c_{p} c(\Omega)\|\boldsymbol{u}\|_{1, \Omega}\right\}|\widetilde{\varphi}-\widetilde{\psi}|_{1, \Omega}^{2} \\
& \geq c_{p}^{-2}\left\{\vartheta_{1}-c_{p} c(\Omega)\|\boldsymbol{u}\|_{1, \Omega}\right\}\|\widetilde{\varphi}-\widetilde{\psi}\|_{1, \Omega}^{2},
\end{aligned}
$$

which shows that $A_{\boldsymbol{u}}$ is strongly monotone with constant $\widetilde{\alpha}_{\boldsymbol{u}}:=c_{p}^{-2}\left\{\vartheta_{1}-c_{p} c(\Omega)\|\boldsymbol{u}\|_{1, \Omega}\right\}$. In turn, proceeding similarly, we find that for all $\widetilde{\varphi}, \widetilde{\psi}, \widetilde{\rho} \in \mathrm{H}_{\Gamma_{\mathrm{D}}}^{1}(\Omega)$ there holds

$$
\begin{aligned}
\mid A_{\boldsymbol{u}}(\widetilde{\varphi}, \widetilde{\rho}) & -A_{\boldsymbol{u}}(\widetilde{\psi}, \widetilde{\rho})|=| \int_{\Omega}\{\vartheta(|\nabla \widetilde{\varphi}|) \nabla \widetilde{\varphi}-\vartheta(|\nabla \widetilde{\psi}|) \nabla \widetilde{\psi}\} \cdot \nabla \widetilde{\rho}-\int_{\Omega}(\widetilde{\varphi}-\widetilde{\psi}) \boldsymbol{u} \cdot \nabla \widetilde{\rho} \mid \\
\leq & \widetilde{\vartheta}_{2}|\widetilde{\varphi}-\widetilde{\psi}|_{1, \Omega}|\widetilde{\rho}|_{1, \Omega}+c(\Omega)\|\widetilde{\varphi}-\widetilde{\psi}\|_{1, \Omega}\|\boldsymbol{u}\|_{1, \Omega}|\widetilde{\rho}|_{1, \Omega} \\
\leq & \left\{\widetilde{\vartheta}_{2}+c(\Omega)\|\boldsymbol{u}\|_{1, \Omega}\right\}\|\widetilde{\varphi}-\widetilde{\psi}\|_{1, \Omega}\|\widetilde{\rho}\|_{1, \Omega},
\end{aligned}
$$

which proves that $A_{\boldsymbol{u}}$ is Lipschitz-continuous with constant $\widetilde{L}_{\boldsymbol{u}}:=\widetilde{\vartheta}_{2}+c(\Omega)\|\boldsymbol{u}\|_{1, \Omega}$. Therefore, a direct application of a classical result on the bijectivity of monotone operators (see, e.g. [27], Thm. 3.3.23) implies the 
existence of a unique solution $\widetilde{\phi}:=\widetilde{\mathbf{S}}(\phi, \boldsymbol{u}) \in \mathrm{H}_{\Gamma_{\mathrm{D}}}^{1}(\Omega)$ of (3.11). Moreover, applying the strong monotonicity of $A_{\boldsymbol{u}}$ to $\widetilde{\varphi}=\widetilde{\phi}$ and $\widetilde{\psi}=0$, and noting from $(3.4)$ that $A_{\boldsymbol{u}}(0, \cdot)=0$, we deduce that

$$
\widetilde{\alpha}_{\boldsymbol{u}}\|\widetilde{\phi}\|_{1, \Omega}^{2} \leq A_{\boldsymbol{u}}(\widetilde{\phi}, \widetilde{\phi})=G_{\phi}(\widetilde{\phi})
$$

which gives $\widetilde{\alpha}_{\boldsymbol{u}}\|\widetilde{\phi}\|_{1, \Omega} \leq\left\|G_{\phi}\right\|$. Finally, using the Cauchy-Schwarz's inequality and the upper bound of $\gamma$ (cf. (2.2)), it follows from (3.12) that $\left\|G_{\phi}\right\| \leq \gamma_{2}|\Omega|^{1 / 2}\|\boldsymbol{k}\|+\|g\|_{0, \Omega}$, which yields (3.23) and finishes the proof.

A simple corollary of the above lemma, which removes the dependence on $\boldsymbol{u}$ of the strong monotonicity constant of $A_{\boldsymbol{u}}$ and of the estimate (3.23), is given as follows.

Lemma 3.6. Let $\phi \in \mathrm{H}_{\Gamma_{\mathrm{D}}}^{1}(\Omega)$ and $\boldsymbol{u} \in \mathbf{H}^{1}(\Omega)$ such that $\|\boldsymbol{u}\|_{1, \Omega}<\frac{\vartheta_{1}}{2 c_{p} c(\Omega)}$ (cf. (2.3), (3.1), (3.5)). Then, there exists a unique $\widetilde{\phi}:=\widetilde{\mathbf{S}}(\phi, \boldsymbol{u}) \in \mathrm{H}_{\Gamma_{\mathrm{D}}}^{1}(\Omega)$ solution of (3.11), and there holds

$$
\|\widetilde{\mathbf{S}}(\phi, \boldsymbol{u})\|_{1, \Omega}=\|\widetilde{\phi}\|_{1, \Omega} \leq \frac{2 c_{p}^{2}}{\vartheta_{1}}\left\{\gamma_{2}|\Omega|^{1 / 2}\|\boldsymbol{k}\|+\|g\|_{0, \Omega}\right\} .
$$

Proof. It follows directly from the proof of Lemma 3.5. Note in particular that the strong monotonicity of $A_{\boldsymbol{u}}$ holds with the constant $\widetilde{\alpha}:=\frac{\vartheta_{1}}{2 c_{p}^{2}}$. Further details are omitted.

We end this section by remarking that the restriction on $\|\boldsymbol{u}\|_{1, \Omega}$ in Lemma 3.6 could also have been taken as $\|\boldsymbol{u}\|_{1, \Omega}<\delta \frac{\vartheta_{1}}{c_{p} c(\Omega)}$ with any $\delta \in(0,1)$. However, we have chosen $\delta=\frac{1}{2}$ for simplicity and because it yields a joint maximization of the constant $\widetilde{\alpha}$ and the upper bound for $\|\boldsymbol{u}\|_{1, \Omega}$.

\subsection{Solvability analysis of the fixed point equation}

Having established in the previous section the well-posedness of the uncoupled problems (3.7) and (3.11), which confirms that the operators $\mathbf{S}, \widetilde{\mathbf{S}}$, and $\mathbf{T}$ ( $c f$. Sect. 3.2 ) are well defined, we now address the solvability analysis of the fixed point equation (3.13). For this purpose, in what follows we verify the hypotheses of the Schauder fixed point theorem, which is stated as follows (see, e.g. [11], Thm. 9.12-1(b)).

Theorem 3.7. Let $W$ be a closed and convex subset of a Banach space $X$ and let $T: W \rightarrow W$ be a continuous mapping such that $\overline{T(W)}$ is compact. Then $T$ has at least one fixed point.

We begin the analysis with the following result.

Lemma 3.8. Given $r>0$, we let $W$ be the closed and convex subset of $\mathrm{H}_{\Gamma_{\mathrm{D}}}^{1}(\Omega)$ defined by

$$
W:=\left\{\phi \in \mathrm{H}_{\Gamma_{\mathrm{D}}}^{1}(\Omega):\|\phi\|_{1, \Omega} \leq r\right\},
$$

and assume that the data satisfy

$$
\left\|\boldsymbol{u}_{\mathrm{D}}\right\|_{1 / 2, \Gamma_{\mathrm{D}}}+r\|\boldsymbol{f}\|_{\infty, \Omega}<\frac{\vartheta_{1}}{2 C_{S} c_{p} c(\Omega)} \quad \text { and } \quad \gamma_{2}|\Omega|^{1 / 2}\|\boldsymbol{k}\|+\|g\|_{0, \Omega} \leq \frac{\vartheta_{1} r}{2 c_{p}^{2}}
$$

Then $\mathbf{T}(W) \subseteq W$.

Proof. Given $\phi \in W$, we get from (3.16) (cf. Lem. 3.4) that

$$
\|\mathbf{S}(\phi)\|_{H}=\|(\boldsymbol{\sigma}, \boldsymbol{u})\|_{H} \leq C_{S}\left\{\left\|\boldsymbol{u}_{\mathrm{D}}\right\|_{1 / 2, \Gamma_{\mathrm{D}}}+r\|\boldsymbol{f}\|_{\infty, \Omega}\right\},
$$


and hence, thanks to the first restriction in (3.25), we observe that $\boldsymbol{u}=\mathbf{S}_{2}(\phi)$ satisfies the hypotheses of Lemma 3.6. Moreover, the corresponding estimate (3.24) gives

$$
\|\mathbf{T}(\phi)\|_{1, \Omega}=\|\widetilde{\mathbf{S}}(\phi, \boldsymbol{u})\|_{1, \Omega} \leq \frac{2 c_{p}^{2}}{\vartheta_{1}}\left\{\gamma_{2}|\Omega|^{1 / 2}\|\boldsymbol{k}\|+\|g\|_{0, \Omega}\right\},
$$

which, due to the second inequality in (3.25), proves that $\mathbf{T}(\phi) \in W$, thus finishing the proof.

Next, we aim to prove the continuity and compactness properties of $\mathbf{T}$, which basically will be direct consequences of the following two lemmas providing the continuity of $\mathbf{S}$ and $\widetilde{\mathbf{S}}$, respectively. We remark in advance that a combination of the Cauchy-Schwarz and Hölder inequalities with the further regularity assumption specified by (3.22) plays a key role in the proof of the first result.

Lemma 3.9. There exists a positive constant $C$, depending on $\mu_{1}, \kappa_{1}, \kappa_{2}, L_{\mu}, \alpha$, and $\varepsilon$ (cf. (2.2), (3.6), (3.15), (3.19), (3.22)), such that

$$
\|\mathbf{S}(\phi)-\mathbf{S}(\psi)\|_{H} \leq C\left\{\|\boldsymbol{f}\|_{\infty, \Omega}\|\phi-\psi\|_{0, \Omega}+\left\|\mathbf{S}_{1}(\psi)\right\|_{\varepsilon, \Omega}\|\phi-\psi\|_{\mathrm{L}^{n / \varepsilon}(\Omega)}\right\} \quad \forall \phi, \psi \in \mathrm{H}_{\Gamma_{\mathrm{D}}}^{1}(\Omega) .
$$

Proof. Given $\phi, \psi \in \mathrm{H}_{\Gamma_{\mathrm{D}}}^{1}(\Omega)$, we let $(\boldsymbol{\sigma}, \boldsymbol{u})=\mathbf{S}(\phi)$ and $(\boldsymbol{\zeta}, \boldsymbol{w})=\mathbf{S}(\psi)$, that is

$$
B_{\phi}((\boldsymbol{\sigma}, \boldsymbol{u}),(\boldsymbol{\tau}, \boldsymbol{v}))=F_{\phi}(\boldsymbol{\tau}, \boldsymbol{v}) \quad \text { and } \quad B_{\psi}((\boldsymbol{\zeta}, \boldsymbol{w}),(\boldsymbol{\tau}, \boldsymbol{v}))=F_{\psi}(\boldsymbol{\tau}, \boldsymbol{v}) \quad \forall(\boldsymbol{\tau}, \boldsymbol{v}) \in H .
$$

It follows, using the ellipticity of $B_{\phi}\left(c f\right.$. (3.19)) and then subtracting and adding the expression $F_{\psi}((\boldsymbol{\sigma}, \boldsymbol{u})-$ $(\boldsymbol{\zeta}, \boldsymbol{w}))=B_{\psi}((\boldsymbol{\zeta}, \boldsymbol{w}),(\boldsymbol{\sigma}, \boldsymbol{u})-(\boldsymbol{\zeta}, \boldsymbol{w}))$, that

$$
\begin{gathered}
\alpha\|(\boldsymbol{\sigma}, \boldsymbol{u})-(\boldsymbol{\zeta}, \boldsymbol{w})\|_{H}^{2} \leq B_{\phi}((\boldsymbol{\sigma}, \boldsymbol{u}),(\boldsymbol{\sigma}, \boldsymbol{u})-(\boldsymbol{\zeta}, \boldsymbol{w}))-B_{\phi}((\boldsymbol{\zeta}, \boldsymbol{w}),(\boldsymbol{\sigma}, \boldsymbol{u})-(\boldsymbol{\zeta}, \boldsymbol{w})) \\
=\left(F_{\phi}-F_{\psi}\right)((\boldsymbol{\sigma}, \boldsymbol{u})-(\boldsymbol{\zeta}, \boldsymbol{w}))+\left(B_{\psi}-B_{\phi}\right)((\boldsymbol{\zeta}, \boldsymbol{w}),(\boldsymbol{\sigma}, \boldsymbol{u})-(\boldsymbol{\zeta}, \boldsymbol{w})) .
\end{gathered}
$$

Then, according to the definition of $F_{\phi}(c f .(3.9))$, and applying the Cauchy-Schwarz's inequality, we deduce that

$$
\begin{gathered}
\left|\left(F_{\phi}-F_{\psi}\right)((\boldsymbol{\sigma}, \boldsymbol{u})-(\boldsymbol{\zeta}, \boldsymbol{w}))\right|=\left|\int_{\Omega} \boldsymbol{f}(\phi-\psi) \cdot(\boldsymbol{u}-\boldsymbol{w})-\kappa_{2} \int_{\Omega} \boldsymbol{f}(\phi-\psi) \cdot \operatorname{div}(\boldsymbol{\sigma}-\boldsymbol{\zeta})\right| \\
\leq\|\boldsymbol{f}\|_{\infty, \Omega}\|\phi-\psi\|_{0, \Omega}\left\{\|\boldsymbol{u}-\boldsymbol{w}\|_{0, \Omega}+\kappa_{2}\|\operatorname{div}(\boldsymbol{\sigma}-\boldsymbol{\zeta})\|_{0, \Omega}\right\} \\
\leq\left(1+\kappa_{2}^{2}\right)^{1 / 2}\|\boldsymbol{f}\|_{\infty, \Omega}\|\phi-\psi\|_{0, \Omega}\|(\boldsymbol{\sigma}, \boldsymbol{u})-(\boldsymbol{\zeta}, \boldsymbol{w})\|_{H} .
\end{gathered}
$$

In turn, it follows easily from (3.8) that

$$
\left(B_{\psi}-B_{\phi}\right)((\boldsymbol{\zeta}, \boldsymbol{w}),(\boldsymbol{\sigma}, \boldsymbol{u})-(\boldsymbol{\zeta}, \boldsymbol{w}))=\int_{\Omega}\left\{\frac{\mu(\phi)-\mu(\psi)}{\mu(\phi) \mu(\psi)}\right\} \boldsymbol{\zeta}^{\mathrm{d}}:\left\{(\boldsymbol{\sigma}-\boldsymbol{\zeta})^{\mathrm{d}}-\kappa_{1} \nabla(\boldsymbol{u}-\boldsymbol{w})\right\},
$$

from which, thanks to the lower bound of $\mu$ (cf. (2.2)) and its Lipschitz-continuity assumption (3.15), and applying Cauchy-Schwarz and Hölder inequalities, we find that

$$
\begin{aligned}
\mid\left(B_{\psi}\right. & \left.-B_{\phi}\right)((\boldsymbol{\zeta}, \boldsymbol{w}),(\boldsymbol{\sigma}, \boldsymbol{u})-(\boldsymbol{\zeta}, \boldsymbol{w}))\left|\leq \frac{L_{\mu}}{\mu_{1}^{2}} \int_{\Omega}\right|(\phi-\psi) \boldsymbol{\zeta}^{\mathrm{d}}||(\boldsymbol{\sigma}-\boldsymbol{\zeta})^{\mathrm{d}}-\kappa_{1} \nabla(\boldsymbol{u}-\boldsymbol{w}) \mid \\
& \leq \frac{L_{\mu}}{\mu_{1}^{2}}\|(\phi-\psi) \boldsymbol{\zeta}\|_{0, \Omega}\left\|(\boldsymbol{\sigma}-\boldsymbol{\zeta})^{\mathrm{d}}-\kappa_{1} \nabla(\boldsymbol{u}-\boldsymbol{w})\right\|_{0, \Omega} \\
& \leq \frac{L_{\mu}}{\mu_{1}^{2}}\|\boldsymbol{\zeta}\|_{\mathbb{L}^{2 p}(\Omega)}\|\phi-\psi\|_{\mathrm{L}^{2 q}(\Omega)}\left\{\|\boldsymbol{\sigma}-\boldsymbol{\zeta}\|_{0, \Omega}+\kappa_{1}|\boldsymbol{u}-\boldsymbol{w}|_{1, \Omega}\right\} \\
& \leq \frac{L_{\mu}\left(1+\kappa_{1}^{2}\right)^{1 / 2}}{\mu_{1}^{2}}\|\boldsymbol{\zeta}\|_{\mathbb{L}^{2 p}(\Omega)}\|\phi-\psi\|_{\mathrm{L}^{2 q}(\Omega)}\|(\boldsymbol{\sigma}, \boldsymbol{u})-(\boldsymbol{\zeta}, \boldsymbol{w})\|_{H},
\end{aligned}
$$


where $p, q \in[1,+\infty)$ are such that $\frac{1}{p}+\frac{1}{q}=1$. Next, bearing in mind the further regularity $\varepsilon$ assumed in (3.22), we notice that the Sobolev embedding Theorem (cf. [1], Thm. 4.12, [29], Thm. 1.3.4) establishes the continuous injection $i_{\varepsilon}: \mathrm{H}^{\varepsilon}(\Omega) \longrightarrow \mathrm{L}^{\varepsilon^{*}}(\Omega)$ with boundedness constant $C_{\varepsilon}$, where

$$
\varepsilon^{*}:= \begin{cases}\frac{2}{1-\varepsilon} & \text { if } n=2, \\ \frac{6}{3-2 \varepsilon} & \text { if } n=3 .\end{cases}
$$

Thus, choosing $p$ such that $2 p=\varepsilon^{*}$, we deduce that $\boldsymbol{\zeta}:=\mathbf{S}_{1}(\psi)$ does in fact belong to $\mathbb{L}^{2 p}(\Omega)$, and hence, thanks to the aforementioned continuity, there holds

$$
\|\boldsymbol{\zeta}\|_{\mathbb{L}^{2 p}(\Omega)} \leq C_{\varepsilon}\|\boldsymbol{\zeta}\|_{\varepsilon, \Omega}
$$

which, when needed, can be bounded by (3.22), yielding for each $\psi$ with $\|\psi\|_{1, \Omega} \leq r$

$$
\|\boldsymbol{\zeta}\|_{\mathbb{L}^{2 p}(\Omega)} \leq C_{\varepsilon} \widetilde{C}_{S}(r)\left\{\left\|\boldsymbol{u}_{\mathrm{D}}\right\|_{1 / 2+\varepsilon, \Gamma_{\mathrm{D}}}+\|\boldsymbol{f}\|_{\infty, \Omega}\|\psi\|_{0, \Omega}\right\} .
$$

In addition, according to the above choice of $p$, that is $p=\varepsilon^{*} / 2$, we readily find that

$$
2 q:=\frac{2 p}{p-1}=\left\{\begin{array}{ll}
\frac{2}{\varepsilon} & \text { if } n=2, \\
\frac{3}{\varepsilon} & \text { if } n=3
\end{array}=\frac{n}{\varepsilon} .\right.
$$

In this way, inequalities (3.27), (3.28), (3.29), and (3.30), together with the identity (3.31), imply (3.26) and complete the proof.

Lemma 3.10. Let $\widetilde{\alpha}:=\frac{\vartheta_{1}}{2 c_{p}^{2}}$ be the strong monotonicity constant provided in the proof of Lemma 3.6. Then, there exists a positive constant $\widetilde{C}$, depending on $\widetilde{\alpha}, c(\Omega)$, and $L_{\gamma}(c f .(3.5),(3.14))$, such that for all $(\phi, \boldsymbol{u}),(\varphi, \boldsymbol{w}) \in \mathrm{H}_{\Gamma_{\mathrm{D}}}^{1}(\Omega) \times \mathbf{H}^{1}(\Omega)$, with $\|\boldsymbol{u}\|_{1, \Omega},\|\boldsymbol{w}\|_{1, \Omega}<\frac{\vartheta_{1}}{2 c_{p} c(\Omega)}$, there holds

$$
\|\widetilde{\mathbf{S}}(\phi, \boldsymbol{u})-\widetilde{\mathbf{S}}(\varphi, \boldsymbol{w})\|_{1, \Omega} \leq \widetilde{C}\left\{\|\boldsymbol{k}\|\|\phi-\varphi\|_{0, \Omega}+\|\widetilde{\mathbf{S}}(\varphi, \boldsymbol{w})\|_{1, \Omega}\|\boldsymbol{u}-\boldsymbol{w}\|_{1, \Omega}\right\} .
$$

Proof. Given $(\phi, \boldsymbol{u}),(\varphi, \boldsymbol{w})$ as stated, we let $\widetilde{\phi}:=\widetilde{\mathbf{S}}(\phi, \boldsymbol{u})$ and $\widetilde{\varphi}:=\widetilde{\mathbf{S}}(\varphi, \boldsymbol{w})$, that is $(c f .(3.11))$

$$
A_{\boldsymbol{u}}(\widetilde{\phi}, \widetilde{\psi})=G_{\phi}(\widetilde{\psi}) \quad \text { and } \quad A_{\boldsymbol{w}}(\widetilde{\varphi}, \widetilde{\psi})=G_{\varphi}(\widetilde{\psi}) \quad \forall \widetilde{\psi} \in \mathrm{H}_{\Gamma_{\mathrm{D}}}^{1}(\Omega) .
$$

It follows, according to the strong monotonicity of $A_{\boldsymbol{u}}$ with constant $\widetilde{\alpha}$, and then subtracting and adding $G_{\varphi}(\widetilde{\phi}-\widetilde{\varphi})=A_{w}(\widetilde{\varphi}, \widetilde{\phi}-\widetilde{\varphi})$, that

$$
\begin{aligned}
\widetilde{\alpha} \| \widetilde{\phi} & -\widetilde{\varphi} \|_{1, \Omega}^{2} \leq A_{\boldsymbol{u}}(\widetilde{\phi}, \widetilde{\phi}-\widetilde{\varphi})-A_{\boldsymbol{u}}(\widetilde{\varphi}, \widetilde{\phi}-\widetilde{\varphi}) \\
& =G_{\phi}(\widetilde{\phi}-\widetilde{\varphi})-G_{\varphi}(\widetilde{\phi}-\widetilde{\varphi})+A_{\boldsymbol{w}}(\widetilde{\varphi}, \widetilde{\phi}-\widetilde{\varphi})-A_{\boldsymbol{u}}(\widetilde{\varphi}, \widetilde{\phi}-\widetilde{\varphi}) \\
& =\int_{\Omega}(\gamma(\phi)-\gamma(\varphi)) \boldsymbol{k} \cdot \nabla(\widetilde{\phi}-\widetilde{\varphi})+\int_{\Omega} \widetilde{\varphi}(\boldsymbol{u}-\boldsymbol{w}) \cdot \nabla(\widetilde{\phi}-\widetilde{\varphi}),
\end{aligned}
$$

where the last equality has employed the definitions given by (3.4) and (3.12). Then, applying the Lipschitzcontinuity of $\gamma(c f$. (3.14)), the Cauchy-Schwarz's inequality, and the estimate (3.5), we deduce from the foregoing equation that

$$
\widetilde{\alpha}\|\widetilde{\phi}-\widetilde{\varphi}\|_{1, \Omega}^{2} \leq\left\{L_{\gamma}\|\boldsymbol{k}\|\|\phi-\varphi\|_{0, \Omega}+c(\Omega)\|\widetilde{\varphi}\|_{1, \Omega}\|\boldsymbol{u}-\boldsymbol{w}\|_{1, \Omega}\right\}|\widetilde{\phi}-\widetilde{\varphi}|_{1, \Omega}
$$

which gives (3.32) and finishes the proof.

The following result is a straightforward corollary of Lemmas 3.9 and 3.10. 
Lemma 3.11. Given $r>0$, we let $W:=\left\{\phi \in \mathrm{H}_{\Gamma_{\mathrm{D}}}^{1}(\Omega):\|\phi\|_{1, \Omega} \leq r\right\}$, and assume that

$$
\left\|\boldsymbol{u}_{\mathrm{D}}\right\|_{1 / 2, \Gamma_{\mathrm{D}}}+r\|\boldsymbol{f}\|_{\infty, \Omega}<\frac{\vartheta_{1}}{2 C_{S} c_{p} c(\Omega)} \quad \text { and } \quad \gamma_{2}|\Omega|^{1 / 2}\|\boldsymbol{k}\|+\|g\|_{0, \Omega} \leq \frac{\vartheta_{1} r}{2 c_{p}^{2}}
$$

Then, with the constants $C$ and $\widetilde{C}$ from Lemmas 3.9 and 3.10 , for all $\phi, \varphi \in \mathrm{H}_{\Gamma_{\mathrm{D}}}^{1}(\Omega)$ there holds

$$
\begin{gathered}
\|\mathbf{T}(\phi)-\mathbf{T}(\varphi)\|_{1, \Omega} \leq\left\{\widetilde{C}\|\boldsymbol{k}\|+C \widetilde{C}\|\mathbf{T}(\varphi)\|_{1, \Omega}\|\boldsymbol{f}\|_{\infty, \Omega}\right\}\|\phi-\varphi\|_{0, \Omega} \\
+C \widetilde{C}\|\mathbf{T}(\varphi)\|_{1, \Omega}\left\|\mathbf{S}_{1}(\varphi)\right\|_{\varepsilon, \Omega}\|\phi-\varphi\|_{\mathrm{L}^{n / \varepsilon}(\Omega)} .
\end{gathered}
$$

Proof. It suffices to recall from Section 3.2 that $\mathbf{T}(\phi)=\widetilde{\mathbf{S}}\left(\phi, \mathbf{S}_{2}(\phi)\right) \quad \forall \phi \in \mathrm{H}_{\Gamma_{\mathrm{D}}}^{1}(\Omega)$, and then apply Lemmas $3.8,3.9$, and 3.10 .

The announced properties of $\mathbf{T}$ are proved now.

Lemma 3.12. Given $r>0$, we let $W:=\left\{\phi \in \mathrm{H}_{\Gamma_{\mathrm{D}}}^{1}(\Omega):\|\phi\|_{1, \Omega} \leq r\right\}$, and assume that

$$
\left\|\boldsymbol{u}_{\mathrm{D}}\right\|_{1 / 2, \Gamma_{\mathrm{D}}}+r\|\boldsymbol{f}\|_{\infty, \Omega}<\frac{\vartheta_{1}}{2 C_{S} c_{p} c(\Omega)} \quad \text { and } \quad \gamma_{2}|\Omega|^{1 / 2}\|\boldsymbol{k}\|+\|g\|_{0, \Omega} \leq \frac{\vartheta_{1} r}{2 c_{p}^{2}}
$$

Then, $\mathbf{T}: W \longrightarrow W$ is continuous and $\overline{\mathbf{T}(W)}$ is compact.

Proof. We first observe, thanks now to the Rellich-Kondrachov compactness Theorem (cf. [1], Thm. 6.3, [29], Thm. 1.3.5), that the injection $i: \mathrm{H}^{1}(\Omega) \longrightarrow \mathrm{L}^{s}(\Omega)$ is compact, and hence continuous, for each $s \geq 1$ (when $n=2$ ), and for each $s \in[1,6)$ (when $n=3$ ). Then, according to the assumptions on the further regularity $\varepsilon$ ( $c f$. (3.22)), namely $\varepsilon \in(0,1)$ in $\mathrm{R}^{2}$ and $\varepsilon \in\left(\frac{1}{2}, 1\right)$ in $\mathrm{R}^{3}$, we realize that $n / \varepsilon$ belongs to the indicated ranges for $s$, and therefore $\mathrm{H}^{1}(\Omega)$ is compactly, and hence continuously, embedded in $\mathrm{L}^{n / \varepsilon}(\Omega)$, which together with (3.33) imply the continuity of $\mathbf{T}$. In turn, let $\left\{\phi_{k}\right\}_{k \in \mathrm{N}}$ be a sequence of $W$, which is clearly bounded. It follows that there exist a subsequence $\left\{\phi_{k}^{(1)}\right\}_{k \in \mathrm{N}} \subseteq\left\{\phi_{k}\right\}_{k \in \mathrm{N}}$ and $\phi \in \mathrm{H}_{\Gamma_{\mathrm{D}}}^{1}(\Omega)$ such that $\phi_{k}^{(1)} \stackrel{w}{\longrightarrow} \phi$. In this way, since the injections $i_{1}: \mathrm{H}_{\Gamma_{\mathrm{D}}}^{1}(\Omega) \longrightarrow \mathrm{L}^{2}(\Omega)$ and $\widetilde{i}_{1}: \mathrm{H}_{\Gamma_{\mathrm{D}}}^{1}(\Omega) \longrightarrow \mathrm{L}^{n / \varepsilon}(\Omega)$ are compact, we deduce that $\phi_{k}^{(1)} \longrightarrow \phi$ in $\mathrm{L}^{2}(\Omega)$ and $\phi_{k}^{(1)} \longrightarrow \phi$ in $\mathrm{L}^{n / \varepsilon}(\Omega)$, which, combined with $(3.33)$, implies that $\mathbf{T}\left(\phi_{k}^{(1)}\right) \longrightarrow \mathbf{T}(\phi)$ in $\mathrm{H}_{\Gamma_{\mathrm{D}}}^{1}(\Omega)$. This proves the compactness of $\overline{\mathbf{T}(W)}$ and finishes the proof.

Finally, the main result of this section is given as follows.

Theorem 3.13. Given $r>0$, we let $W:=\left\{\phi \in \mathrm{H}_{\Gamma_{\mathrm{D}}}^{1}(\Omega):\|\phi\|_{1, \Omega} \leq r\right\}$, and assume that

$$
\left\|\boldsymbol{u}_{\mathrm{D}}\right\|_{1 / 2, \Gamma_{\mathrm{D}}}+r\|\boldsymbol{f}\|_{\infty, \Omega}<\frac{\vartheta_{1}}{2 C_{S} c_{p} c(\Omega)} \quad \text { and } \quad \gamma_{2}|\Omega|^{1 / 2}\|\boldsymbol{k}\|+\|g\|_{0, \Omega} \leq \frac{\vartheta_{1} r}{2 c_{p}^{2}}
$$

Then the augmented mixed-primal problem (3.10) has at least one solution $(\boldsymbol{\sigma}, \boldsymbol{u}, \phi) \in \mathbb{H}_{N}(\mathbf{d i v} ; \Omega) \times \mathbf{H}^{1}(\Omega) \times$ $\mathrm{H}_{\Gamma_{\mathrm{D}}}^{1}(\Omega)$ with $\phi \in W$, and there holds

$$
\|\phi\|_{1, \Omega} \leq \frac{2 c_{p}^{2}}{\vartheta_{1}}\left\{\gamma_{2}|\Omega|^{1 / 2}\|\boldsymbol{k}\|+\|g\|_{0, \Omega}\right\}
$$

and

$$
\|(\boldsymbol{\sigma}, \boldsymbol{u})\|_{H} \leq C_{S}\left\{\left\|\boldsymbol{u}_{\mathrm{D}}\right\|_{1 / 2, \Gamma_{\mathrm{D}}}+\|\boldsymbol{f}\|_{\infty, \Omega}\|\phi\|_{1, \Omega}\right\} .
$$


Moreover, if the data $\boldsymbol{k}, \boldsymbol{f}$, and $\boldsymbol{u}_{\mathrm{D}}$ are sufficiently small so that, with the constants $C, \widetilde{C}$, and $\widetilde{C}_{S}(r)$ from Lemmas 3.9 and 3.10 , and estimate (3.22), and denoting by $\widetilde{C}_{\varepsilon}$ the boundedness constant of the continuous injection of $\mathrm{H}^{1}(\Omega)$ into $\mathrm{L}^{n / \varepsilon}(\Omega)$, there holds

$$
\widetilde{C}\|\boldsymbol{k}\|+C \widetilde{C} r\left\{\left(1+r \widetilde{C}_{\varepsilon} \widetilde{C}_{S}(r)\right)\|\boldsymbol{f}\|_{\infty, \Omega}+\widetilde{C}_{\varepsilon} \widetilde{C}_{S}(r)\left\|\boldsymbol{u}_{\mathrm{D}}\right\|_{1 / 2+\varepsilon, \Gamma_{\mathrm{D}}}\right\}<1,
$$

then the solution $\phi$ is unique in $W$.

Proof. According to the equivalence between (3.10) and the fixed point equation (3.13), and thanks to the previous Lemmas 3.8 and 3.12, the existence of solution is just a straightforward application of the Schauder fixed point theorem ( $c f$. Thm. 3.7). In turn, the estimates (3.35) and (3.36) follow from (3.16) (cf. Lem. 3.4) and (3.24) (cf. Lem. 3.6). Furthermore, given another solution $\varphi \in W$ of (3.13), the estimates $\|\mathbf{T}(\varphi)\|_{1, \Omega}=$ $\|\varphi\|_{1, \Omega} \leq r,\left\|\mathbf{S}_{1}(\varphi)\right\|_{\varepsilon, \Omega} \leq \widetilde{C}_{S}(r)\left\{\left\|\boldsymbol{u}_{\mathrm{D}}\right\|_{1 / 2+\varepsilon, \Gamma_{\mathrm{D}}}+\|\boldsymbol{f}\|_{\infty, \Omega}\|\varphi\|_{1, \Omega}\right\}(c f .(3.22))$, and $\|\psi\|_{\mathrm{L}^{n / \varepsilon}(\Omega)} \leq \widetilde{C}_{\varepsilon}\|\psi\|_{1, \Omega}$ $\forall \psi \in \mathrm{H}^{1}(\Omega)$, confirm (3.37) as a sufficient condition for concluding, together with (3.33), that $\phi=\varphi$.

It is important to highlight here that the uniqueness of $\phi$ certainly implies, according to Lemma 3.4, the uniqueness of the solution $\mathbf{S}(\phi):=(\boldsymbol{\sigma}, \boldsymbol{u}) \in H$ of problem (3.7), and hence the foregoing theorem actually guarantees that, under the asumption (3.34) on the data, there exists a unique solution $(\boldsymbol{\sigma}, \boldsymbol{u}, \phi) \in \mathbb{H}_{N}(\operatorname{div} ; \Omega) \times$ $\mathbf{H}^{1}(\Omega) \times \mathrm{H}_{\Gamma_{\mathrm{D}}}^{1}(\Omega)$ of problem (3.10) such that $\phi \in W$.

\section{The Galerkin scheme}

In this section we introduce and analyze the Galerkin scheme of the augmented mixed-primal problem (3.10). To this end, we now let $\mathcal{T}_{h}$ be a regular triangulation of $\Omega$ by triangles $K$ (resp. tetrahedra $K$ in $\mathrm{R}^{3}$ ) of diameter $h_{K}$, and define the meshsize $h:=\max \left\{h_{K}: \quad K \in \mathcal{T}_{h}\right\}$. In addition, given an integer $k \geq 0$, for each $K \in \mathcal{T}_{h}$ we let $\mathrm{P}_{k}(K)$ be the space of polynomial functions on $K$ of degree $\leq k$, and define the corresponding local Raviart-Thomas space of order $k$ as

$$
\mathrm{RT}_{k}(K):=\mathbf{P}_{k}(K) \oplus \mathrm{P}_{k}(K) \boldsymbol{x}
$$

where, according to the notations described in Section 1, $\mathbf{P}_{k}(K)=\left[\mathrm{P}_{k}(K)\right]^{n}$, and $\boldsymbol{x}$ is the generic vector in $\mathrm{R}^{n}$. Then, we introduce the finite element subspaces approximating the unknowns $\boldsymbol{\sigma}, \boldsymbol{u}$, and $\phi$, respectively, as the global Raviart-Thomas space of order $k$, and the corresponding Lagrange spaces given by the continuous piecewise polynomials of degree $\leq k+1$, that is

$$
\begin{gathered}
\mathbb{H}_{h}^{\boldsymbol{\sigma}}:=\left\{\boldsymbol{\tau}_{h} \in \mathbb{H}_{N}(\operatorname{div} ; \Omega):\left.\quad \mathbf{c}^{\mathrm{t}} \boldsymbol{\tau}_{h}\right|_{K} \in \mathrm{RT}_{k}(K) \quad \forall \mathbf{c} \in \mathrm{R}^{n}, \quad \forall K \in \mathcal{T}_{h}\right\}, \\
\mathbf{H}_{h}^{u}:=\left\{\boldsymbol{v}_{h} \in \mathbf{C}(\Omega):\left.\quad \boldsymbol{v}_{h}\right|_{K} \in \mathbf{P}_{k+1}(K) \quad \forall K \in \mathcal{T}_{h}\right\}, \\
\mathrm{H}_{h}^{\phi}:=\left\{\psi_{h} \in C(\Omega) \cap \mathrm{H}_{\Gamma_{\mathrm{D}}}^{1}(\Omega):\left.\quad \psi_{h}\right|_{K} \in \mathrm{P}_{k+1}(K) \quad \forall K \in \mathcal{T}_{h}\right\} .
\end{gathered}
$$

In this way, the underlying Galerkin's scheme, given by the discrete counterpart of (3.10), reads: find $\left(\boldsymbol{\sigma}_{h}, \boldsymbol{u}_{h}, \phi_{h}\right) \in \mathbb{H}_{h}^{\boldsymbol{\sigma}} \times \mathbf{H}_{h}^{u} \times \mathrm{H}_{h}^{\phi}$ such that

$$
\begin{gathered}
B_{\phi_{h}}\left(\left(\boldsymbol{\sigma}_{h}, \boldsymbol{u}_{h}\right),\left(\boldsymbol{\tau}_{h}, \boldsymbol{v}_{h}\right)\right)=F_{\phi_{h}}\left(\boldsymbol{\tau}_{h}, \boldsymbol{v}_{h}\right) \quad \forall\left(\boldsymbol{\tau}_{h}, \boldsymbol{v}_{h}\right) \in \mathbb{H}_{h}^{\boldsymbol{\sigma}} \times \mathbf{H}_{h}^{\boldsymbol{u}}, \\
A_{\boldsymbol{u}_{h}}\left(\phi_{h}, \psi_{h}\right)=\int_{\Omega} \gamma\left(\phi_{h}\right) \boldsymbol{k} \cdot \nabla \psi_{h}+\int_{\Omega} g \psi_{h} \quad \forall \psi_{h} \in \mathrm{H}_{h}^{\phi} .
\end{gathered}
$$


Throughout the rest of this section we adopt the discrete analogue of the fixed point strategy introduced in Section 3.3. Hence, we now let $\mathbf{S}_{h}: \mathrm{H}_{h}^{\phi} \longrightarrow \mathbb{H}_{h}^{\boldsymbol{\sigma}} \times \mathbf{H}_{h}^{\boldsymbol{u}}$ be the operator defined by

$$
\mathbf{S}_{h}\left(\phi_{h}\right)=\left(\mathbf{S}_{1, h}\left(\phi_{h}\right), \mathbf{S}_{2, h}\left(\phi_{h}\right)\right):=\left(\boldsymbol{\sigma}_{h}, \boldsymbol{u}_{h}\right) \quad \forall \phi_{h} \in \mathrm{H}_{h}^{\phi},
$$

where $\left(\boldsymbol{\sigma}_{h}, \boldsymbol{u}_{h}\right) \in \mathbb{H}_{h}^{\boldsymbol{\sigma}} \times \mathbf{H}_{h}^{\boldsymbol{u}}$ is the unique solution of

$$
B_{\phi_{h}}\left(\left(\boldsymbol{\sigma}_{h}, \boldsymbol{u}_{h}\right),\left(\boldsymbol{\tau}_{h}, \boldsymbol{v}_{h}\right)\right)=F_{\phi_{h}}\left(\boldsymbol{\tau}_{h}, \boldsymbol{v}_{h}\right) \quad \forall\left(\boldsymbol{\tau}_{h}, \boldsymbol{v}_{h}\right) \in \mathbb{H}_{h}^{\boldsymbol{\sigma}} \times \mathbf{H}_{h}^{u},
$$

with $B_{\phi_{h}}$ and $F_{\phi_{h}}$ being defined by (3.8) and (3.9), respectively, with $\phi=\phi_{h}$. In addition, we let $\widetilde{\mathbf{S}}_{h}: \mathrm{H}_{h}^{\phi} \times \mathbf{H}_{h}^{u} \longrightarrow$ $\mathrm{H}_{h}^{\phi}$ be the operator defined by

$$
\widetilde{\mathbf{S}}_{h}\left(\phi_{h}, \boldsymbol{u}_{h}\right):=\widetilde{\phi}_{h} \quad \forall\left(\phi_{h}, \boldsymbol{u}_{h}\right) \in \mathrm{H}_{h}^{\phi} \times \mathbf{H}_{h}^{u},
$$

where $\widetilde{\phi}_{h} \in \mathrm{H}_{h}^{\phi}$ is the unique solution of

$$
A_{\boldsymbol{u}_{h}}\left(\widetilde{\phi}_{h}, \widetilde{\psi}_{h}\right)=G_{\phi_{h}}\left(\widetilde{\psi}_{h}\right) \quad \forall \widetilde{\psi}_{h} \in \mathrm{H}_{h}^{\phi},
$$

with $A_{\boldsymbol{u}_{h}}$ and $G_{\phi_{h}}$ being defined by (3.4) and (3.12), respectively, with $\boldsymbol{u}=\boldsymbol{u}_{h}$ and $\phi=\phi_{h}$. Finally, we define the operator $\mathbf{T}_{h}: \mathrm{H}_{h}^{\phi} \longrightarrow \mathrm{H}_{h}^{\phi}$ by

$$
\mathbf{T}_{h}\left(\phi_{h}\right):=\widetilde{\mathbf{S}}_{h}\left(\phi_{h}, \mathbf{S}_{2, h}\left(\phi_{h}\right)\right) \quad \forall \phi_{h} \in \mathrm{H}_{h}^{\phi},
$$

and realize that (4.4) can be rewritten, equivalently, as: find $\phi_{h} \in \mathrm{H}_{h}^{\phi}$ such that

$$
\mathbf{T}_{h}\left(\phi_{h}\right)=\phi_{h} .
$$

Certainly, all the above makes sense if we guarantee that the discrete problems (4.5) and (4.6) are well-posed. Indeed, it is easy to see that the respective proofs are almost verbatim of the continuous analogues provided in Section 3.3, and hence we simply state the corresponding results as follows.

Lemma 4.1. Assume that $\kappa_{1} \in\left(0, \frac{2 \delta \mu_{1}}{\mu_{2}}\right)$ with $\delta \in\left(0,2 \mu_{1}\right)$, and that $0<\kappa_{2}, \kappa_{3}$. Then, for each $\phi_{h} \in \mathrm{H}_{h}^{\phi}$ the problem (4.5) has a unique solution $\mathbf{S}_{h}\left(\phi_{h}\right):=\left(\boldsymbol{\sigma}_{h}, \boldsymbol{u}_{h}\right) \in \mathbb{H}_{h}^{\boldsymbol{\sigma}} \times \mathbf{H}_{h}^{u}$. Moreover, with the same constant $C_{S}>0$ from Lemma 3.4, there holds

$$
\left\|\mathbf{S}_{h}\left(\phi_{h}\right)\right\|_{H}=\left\|\left(\boldsymbol{\sigma}_{h}, \boldsymbol{u}_{h}\right)\right\|_{H} \leq C_{S}\left\{\left\|\boldsymbol{u}_{\mathrm{D}}\right\|_{1 / 2, \Gamma_{\mathrm{D}}}+\|\boldsymbol{f}\|_{\infty, \Omega}\left\|\phi_{h}\right\|_{0, \Omega}\right\} \quad \forall \phi_{h} \in \mathrm{H}_{h}^{\phi}
$$

Proof. It suffices to see that for each $\phi_{h} \in \mathrm{H}_{h}^{\phi}, B_{\phi_{h}}$ is elliptic on $\mathbb{H}_{h}^{\boldsymbol{\sigma}} \times \mathbf{H}_{h}^{u}$ with the same constant $\alpha$ from Lemma 3.4 (cf. (3.19)), and that $\left\|F_{\phi_{h}}\right\|_{\left(\mathbb{H}_{h}^{\sigma} \times \mathbf{H}_{h}^{u}\right)^{\prime}}$ is bounded as in (3.21) with $\phi_{h}$ in place of $\phi$. The rest of the proof is a direct application of the Lax-Milgram's lemma.

Lemma 4.2. Let $\phi_{h} \in \mathrm{H}_{h}^{\phi}$ and $\boldsymbol{u}_{h} \in \mathbf{H}_{h}^{u}$ such that $\left\|\boldsymbol{u}_{h}\right\|_{1, \Omega}<\frac{\vartheta_{1}}{2 c_{p} c(\Omega)}$ (cf. (2.3), (3.1), (3.5)). Then, there exists a unique $\widetilde{\phi}_{h}:=\widetilde{\mathbf{S}}_{h}\left(\phi_{h}, \boldsymbol{u}_{h}\right) \in \mathrm{H}_{h}^{\phi}$ solution of (4.6), and there holds

$$
\left\|\widetilde{\mathbf{S}}_{h}\left(\phi_{h}, \boldsymbol{u}_{h}\right)\right\|_{1, \Omega}=\left\|\widetilde{\phi}_{h}\right\|_{1, \Omega} \leq \frac{2 c_{p}^{2}}{\vartheta_{1}}\left\{\gamma_{2}|\Omega|^{1 / 2}\|\boldsymbol{k}\|+\|g\|_{0, \Omega}\right\} .
$$

Proof. It basically follows by observing that, under the assumption on $\left\|\boldsymbol{u}_{h}\right\|_{1, \Omega}, A_{\boldsymbol{u}_{h}}$ becomes Lipschitzcontinuous and strongly monotone on $\mathrm{H}_{h}^{\phi} \times \mathrm{H}_{h}^{\phi}$ with the constants $\widetilde{L}_{\boldsymbol{u}_{h}}:=\widetilde{\vartheta}_{2}+c(\Omega)\left\|\boldsymbol{u}_{h}\right\|_{1, \Omega}$ and $\widetilde{\alpha}:=\frac{\vartheta_{1}}{2 c_{p}^{2}}$ given in the proofs of Lemmas 3.5 and 3.6, respectively, and then applying again ([27], Thm. 3.3.23). In addition, the fact that $\left\|G_{\phi}\right\|$ is bounded independently of $\phi$ ( $c f$. Proof of Lem. 3.5), confirms the same upper bound for $\left\|G_{\phi_{h}}\right\|_{\left(\mathrm{H}_{h}^{\phi}\right)^{\prime}}$. 
We now aim to show the solvability of (4.4) by analyzing the equivalent fixed point equation (4.7). To this end, in what follows we verify the hypotheses of the Brouwer fixed point theorem, which is given as follows (see, e.g. [11], Thm. 9.9-2).

Theorem 4.3. Let $W$ be a compact and convex subset of a finite dimensional Banach space $X$ and let $T$ : $W \rightarrow W$ be a continuous mapping. Then $T$ has at least one fixed point.

We begin with the discrete version of Lemma 3.8 .

Lemma 4.4. Given $r>0$, we let $W_{h}:=\left\{\phi_{h} \in \mathrm{H}_{h}^{\phi}: \quad\left\|\phi_{h}\right\|_{1, \Omega} \leq r\right\}$, and assume that

$$
\left\|\boldsymbol{u}_{\mathrm{D}}\right\|_{1 / 2, \Gamma_{\mathrm{D}}}+r\|\boldsymbol{f}\|_{\infty, \Omega}<\frac{\vartheta_{1}}{2 C_{S} c_{p} c(\Omega)} \quad \text { and } \quad \gamma_{2}|\Omega|^{1 / 2}\|\boldsymbol{k}\|+\|g\|_{0, \Omega} \leq \frac{\vartheta_{1} r}{2 c_{p}^{2}}
$$

Then $\mathbf{T}_{h}\left(W_{h}\right) \subseteq W_{h}$.

Proof. It is a straightforward consequence of Lemmas 4.1 and 4.2 .

The discrete analogue of Lemma 3.9 is provided next. We notice in advance that, instead of the regularity assumption employed in the proof of that result, which actually is not needed nor could be applied in the present discrete case, we simply utilize a $\mathrm{L}^{4}-\mathrm{L}^{4}-\mathrm{L}^{2}$ argument.

Lemma 4.5. There exists a positive constant $C$, depending on $\mu_{1}, \kappa_{1}, \kappa_{2}, L_{\mu}$, and $\alpha$ (cf. (2.2), (3.6), (3.15), (3.19)), such that

$$
\left\|\mathbf{S}_{h}\left(\phi_{h}\right)-\mathbf{S}_{h}\left(\psi_{h}\right)\right\|_{H} \leq C\left\{\|\boldsymbol{f}\|_{\infty, \Omega}\left\|\phi_{h}-\psi_{h}\right\|_{0, \Omega}+\left\|\mathbf{S}_{1, h}\left(\psi_{h}\right)\right\|_{\mathbb{L}^{4}(\Omega)}\left\|\phi_{h}-\psi_{h}\right\|_{L^{4}(\Omega)}\right\},
$$

for all $\phi_{h}, \psi_{h} \in \mathrm{H}_{h}^{\phi}$.

Proof. It proceeds exactly as in the proof of Lemma 3.9, except for the derivation of the discrete analogue of (3.29), where, instead of choosing the values of $p$ and $q$ determined by the regularity parameter $\delta$, it suffices to take $p=q=2$, thus obtaining

$$
\begin{aligned}
& \left|\left(B_{\psi_{h}}-B_{\phi_{h}}\right)\left(\left(\boldsymbol{\zeta}_{h}, \boldsymbol{w}_{h}\right),\left(\boldsymbol{\sigma}_{h}, \boldsymbol{u}_{h}\right)-\left(\boldsymbol{\zeta}_{h}, \boldsymbol{w}_{h}\right)\right)\right| \\
& \quad \leq \frac{L_{\mu}\left(1+\kappa_{1}^{2}\right)^{1 / 2}}{\mu_{1}^{2}}\left\|\boldsymbol{\zeta}_{h}\right\|_{L^{4}(\Omega)}\left\|\phi_{h}-\psi_{h}\right\|_{L^{4}(\Omega)}\left\|\left(\boldsymbol{\sigma}_{h}, \boldsymbol{u}_{h}\right)-\left(\boldsymbol{\zeta}_{h}, \boldsymbol{w}_{h}\right)\right\|_{H},
\end{aligned}
$$

for all $\phi_{h}, \psi_{h} \in \mathrm{H}_{h}^{\phi}$, with $\left(\boldsymbol{\sigma}_{h}, \boldsymbol{u}_{h}\right):=\mathbf{S}_{h}\left(\phi_{h}\right)$ and $\left(\boldsymbol{\zeta}_{h}, \boldsymbol{w}_{h}\right):=\mathbf{S}_{h}\left(\psi_{h}\right)$. Thus, the fact that the elements of $\mathbb{H}_{h}^{\boldsymbol{\sigma}}$ are piecewise polynomials insures that $\left\|\boldsymbol{\zeta}_{h}\right\|_{\mathbb{L}^{4}(\Omega)}<+\infty$ for each $\boldsymbol{\zeta}_{h} \in \mathbb{H}_{h}^{\sigma}$. Further details are omitted.

Now, utilizing Lemma 4.5 and the discrete analogue of Lemma 3.10 (which for sake of space saving is not specified here), we can prove the discrete version of Lemma 3.11.

Lemma 4.6. Given $r>0$, we let $W_{h}:=\left\{\phi_{h} \in \mathrm{H}_{h}^{\phi}: \quad\left\|\phi_{h}\right\|_{1, \Omega} \leq r\right\}$, and assume that

$$
\left\|\boldsymbol{u}_{\mathrm{D}}\right\|_{1 / 2, \Gamma_{\mathrm{D}}}+r\|\boldsymbol{f}\|_{\infty, \Omega}<\frac{\vartheta_{1}}{2 C_{S} c_{p} c(\Omega)} \quad \text { and } \quad \gamma_{2}|\Omega|^{1 / 2}\|\boldsymbol{k}\|+\|g\|_{0, \Omega} \leq \frac{\vartheta_{1} r}{2 c_{p}^{2}}
$$

Then, with the constants $C$ and $\widetilde{C}$ from Lemmas 4.5 and 3.10 , for all $\phi_{h}, \varphi_{h} \in \mathrm{H}_{h}^{\phi}$ there holds

$$
\begin{gathered}
\left\|\mathbf{T}_{h}\left(\phi_{h}\right)-\mathbf{T}_{h}\left(\varphi_{h}\right)\right\|_{1, \Omega} \leq\left\{\widetilde{C}\|\boldsymbol{k}\|+C \widetilde{C}\left\|\mathbf{T}_{h}\left(\varphi_{h}\right)\right\|_{1, \Omega}\|\boldsymbol{f}\|_{\infty, \Omega}\right\}\left\|\phi_{h}-\varphi_{h}\right\|_{0, \Omega} \\
+C \widetilde{C}\left\|\mathbf{T}_{h}\left(\varphi_{h}\right)\right\|_{1, \Omega}\left\|\mathbf{S}_{1, h}\left(\varphi_{h}\right)\right\|_{\mathrm{L}^{4}(\Omega)}\left\|\phi_{h}-\varphi_{h}\right\|_{\mathrm{L}^{4}(\Omega)} .
\end{gathered}
$$


Consequently, since the foregoing lemma and the continuous injection of $\mathrm{H}^{1}(\Omega)$ into $\mathrm{L}^{4}(\Omega)$ confirm the continuity of $\mathbf{T}_{h}$, we conclude, thanks to the Brouwer fixed point theorem (cf. Thm. 4.3) and Lemmas 4.4 and 4.6, the main result of this section.

Theorem 4.7. Given $r>0$, we let $W_{h}:=\left\{\phi_{h} \in \mathrm{H}_{h}^{\phi}:\left\|\phi_{h}\right\|_{1, \Omega} \leq r\right\}$, and assume that

$$
\left\|\boldsymbol{u}_{\mathrm{D}}\right\|_{1 / 2, \Gamma_{\mathrm{D}}}+r\|\boldsymbol{f}\|_{\infty, \Omega}<\frac{\vartheta_{1}}{2 C_{S} c_{p} c(\Omega)} \quad \text { and } \quad \gamma_{2}|\Omega|^{1 / 2}\|\boldsymbol{k}\|+\|g\|_{0, \Omega} \leq \frac{\vartheta_{1} r}{2 c_{p}^{2}}
$$

Then the Galerkin scheme (4.4) has at least one solution $\left(\boldsymbol{\sigma}_{h}, \boldsymbol{u}_{h}, \phi_{h}\right) \in \mathbb{H}_{h}^{\boldsymbol{\sigma}} \times \mathbf{H}_{h}^{u} \times \mathrm{H}_{h}^{\phi}$ with $\phi_{h} \in W_{h}$, and there holds

$$
\left\|\phi_{h}\right\|_{1, \Omega} \leq \frac{2 c_{p}^{2}}{\vartheta_{1}}\left\{\gamma_{2}|\Omega|^{1 / 2}\|\boldsymbol{k}\|+\|g\|_{0, \Omega}\right\}
$$

and

$$
\left\|\left(\boldsymbol{\sigma}_{h}, \boldsymbol{u}_{h}\right)\right\|_{H} \leq C_{S}\left\{\left\|\boldsymbol{u}_{\mathrm{D}}\right\|_{1 / 2, \Gamma_{\mathrm{D}}}+\|\boldsymbol{f}\|_{\infty, \Omega}\left\|\phi_{h}\right\|_{1, \Omega}\right\} .
$$

\section{A PRIORI ERROR ANALYSIS}

Given $(\boldsymbol{\sigma}, \boldsymbol{u}, \phi) \in \mathbb{H}_{N}(\operatorname{div} ; \Omega) \times \mathbf{H}^{1}(\Omega) \times \mathrm{H}_{\Gamma_{\mathrm{D}}}^{1}(\Omega)$ with $\phi \in W$, and $\left(\boldsymbol{\sigma}_{h}, \boldsymbol{u}_{h}, \phi_{h}\right) \in \mathbb{H}_{h}^{\boldsymbol{\sigma}} \times \mathbf{H}_{h}^{\boldsymbol{u}} \times \mathrm{H}_{h}^{\phi}$ with $\phi_{h} \in W_{h}$, solutions of (3.10) and (4.4), respectively, we now aim to derive a corresponding a priori error estimate. For this purpose, we now recall from (3.10) and (4.4), that the above means that

$$
\begin{aligned}
& B_{\phi}((\boldsymbol{\sigma}, \boldsymbol{u}),(\boldsymbol{\tau}, \boldsymbol{v}))=F_{\phi}(\boldsymbol{\tau}, \boldsymbol{v}) \quad \forall(\boldsymbol{\tau}, \boldsymbol{v}) \in \mathbb{H}_{N}(\operatorname{div} ; \Omega) \times \mathbf{H}^{1}(\Omega), \\
& B_{\phi_{h}}\left(\left(\boldsymbol{\sigma}_{h}, \boldsymbol{u}_{h}\right),\left(\boldsymbol{\tau}_{h}, \boldsymbol{v}_{h}\right)\right)=F_{\phi_{h}}\left(\boldsymbol{\tau}_{h}, \boldsymbol{v}_{h}\right) \quad \forall\left(\boldsymbol{\tau}_{h}, \boldsymbol{v}_{h}\right) \in \mathbb{H}_{h}^{\boldsymbol{\sigma}} \times \mathbf{H}_{h}^{\boldsymbol{u}},
\end{aligned}
$$

and

$$
\begin{gathered}
A_{\boldsymbol{u}}(\phi, \psi)=G_{\phi}(\psi) \quad \forall \psi \in \mathrm{H}_{\Gamma_{\mathrm{D}}}^{1}(\Omega), \\
A_{\boldsymbol{u}_{h}}\left(\phi_{h}, \psi_{h}\right)=G_{\phi_{h}}\left(\psi_{h}\right) \quad \forall \psi_{h} \in \mathrm{H}_{h}^{\phi} .
\end{gathered}
$$

Next, we recall from [22] a Strang-type lemma, which will be utilized in our subsequent analysis.

Lemma 5.1. Let $H$ be a Hilbert space, $F \in H^{\prime}$, and $\mathbf{A}: H \rightarrow H^{\prime}$ a nonlinear operator. In addition, let $\left\{H_{n}\right\}_{n \in N}$ be a sequence of finite dimensional subspaces of $H$, and for each $n \in N$ consider a nonlinear operator $\mathbf{A}_{n}: H_{n} \rightarrow H_{n}^{\prime}$ and a functional $F_{n} \in H_{n}^{\prime}$. Assume that the family $\{\mathbf{A}\} \cup\left\{\mathbf{A}_{n}\right\}_{n \in N}$ is uniformly Lipschitz continuous and strongly monotone with constants $\Lambda_{\mathrm{LC}}$ and $\Lambda_{\mathrm{SM}}$, respectively. In turn, let $u \in H$ and $u_{n} \in H_{n}$ such that

$$
[\mathbf{A}(u), v]=[F, v] \quad \forall v \in H \quad \text { and } \quad\left[\mathbf{A}_{n}\left(u_{n}\right), v_{n}\right]=\left[F_{n}, v_{n}\right] \quad \forall v_{n} \in H_{n},
$$

where $[\cdot, \cdot]$ denotes the duality pairings of both $H^{\prime} \times H$ and $H_{n}^{\prime} \times H_{n}$. Then for each $n \in N$ there holds

$$
\begin{aligned}
\left\|u-u_{n}\right\|_{H} \leq \Lambda_{\mathrm{ST}}\left\{\sup _{\substack{w_{n} \in H_{n} \\
w_{n} \neq \mathbf{0}}} \frac{\left|\left[F, w_{n}\right]-\left[F_{n}, w_{n}\right]\right|}{\left\|w_{n}\right\|_{H}}\right. \\
\left.+\inf _{\substack{v_{n} \in H_{n} \\
v_{n} \neq \mathbf{0}}}\left(\left\|u-v_{n}\right\|_{H}+\sup _{\substack{w_{n} \in H_{n} \\
w_{n} \neq \mathbf{0}}} \frac{\left|\left[\mathbf{A}\left(v_{n}\right), w_{n}\right]-\left[\mathbf{A}_{n}\left(v_{n}\right), w_{n}\right]\right|}{\left\|w_{n}\right\|_{H}}\right)\right\},
\end{aligned}
$$

with $\Lambda_{\mathrm{ST}}:=\Lambda_{\mathrm{SM}}^{-1} \max \left\{1, \Lambda_{\mathrm{SM}}+\Lambda_{\mathrm{LC}}\right\}$.

Proof. It is a particular case of ([22], Thm. 6.4). 
We begin our analysis by denoting as usual

$$
\operatorname{dist}\left(\phi, \mathrm{H}_{h}^{\phi}\right):=\inf _{\varphi_{h} \in \mathrm{H}_{h}^{\phi}}\left\|\phi-\varphi_{h}\right\|_{1, \Omega}
$$

and

$$
\operatorname{dist}\left((\boldsymbol{\sigma}, \boldsymbol{u}), \mathbb{H}_{h}^{\boldsymbol{\sigma}} \times \mathbf{H}_{h}^{\boldsymbol{u}}\right):=\inf _{\left(\boldsymbol{\tau}_{h}, \boldsymbol{v}_{h}\right) \in \mathbb{H}_{h}^{\boldsymbol{\sigma}} \times \mathbf{H}_{h}^{u}}\left\|(\boldsymbol{\sigma}, \boldsymbol{u})-\left(\boldsymbol{\tau}_{h}, \boldsymbol{v}_{h}\right)\right\|_{H} .
$$

Then, we have the following result concerning $\left\|\phi-\phi_{h}\right\|_{1, \Omega}$.

Lemma 5.2. Let $\widetilde{C}_{\mathrm{ST}}:=\widetilde{\alpha}^{-1} \max \{1, \widetilde{\alpha}+\widetilde{L}\}$, with $\widetilde{\alpha}:=\frac{\vartheta_{1}}{2 c_{p}^{2}}$ and $\widetilde{L}:=\widetilde{\vartheta}_{2}+\frac{\vartheta_{1}}{2 c_{p}}$. Then there holds

$$
\begin{gathered}
\left\|\phi-\phi_{h}\right\|_{1, \Omega} \leq \widetilde{C}_{\mathrm{ST}}\left\{L_{\gamma}\|\boldsymbol{k}\|\left\|\phi-\phi_{h}\right\|_{0, \Omega}+c(\Omega)\|\phi\|_{1, \Omega}\left\|\boldsymbol{u}-\boldsymbol{u}_{h}\right\|_{1, \Omega}\right. \\
\left.+\quad\left(1+c(\Omega)\left\|\boldsymbol{u}-\boldsymbol{u}_{h}\right\|_{1, \Omega}\right) \operatorname{dist}\left(\phi, \mathrm{H}_{h}^{\phi}\right)\right\} .
\end{gathered}
$$

Proof. We first observe from Lemmas 3.5, 3.6, and 4.2, that the nonlinear operators $A_{\boldsymbol{u}}$ and $A_{\boldsymbol{u}_{h}}$ are both strongly monotone and Lipschitz-continuous on their corresponding spaces with constants $\widetilde{\alpha}$ and $\widetilde{L}$, respectively. Then, by applying the abstract Lemma 5.1 to the context (5.2), we find that

$$
\begin{gathered}
\left\|\phi-\phi_{h}\right\|_{1, \Omega} \leq \widetilde{C}_{\mathrm{ST}}\left\{\sup _{\substack{\psi_{h} \in \mathrm{H}_{h}^{\phi} \\
\psi_{h} \neq \mathbf{0}}} \frac{\left|G_{\phi}\left(\psi_{h}\right)-G_{\phi_{h}}\left(\psi_{h}\right)\right|}{\left\|\psi_{h}\right\|_{1, \Omega}}\right. \\
\left.+\inf _{\substack{\varphi_{h} \in \mathrm{H}_{h}^{\phi} \\
\varphi_{h} \neq \mathbf{0}}}\left(\left\|\phi-\varphi_{h}\right\|_{1, \Omega}+\sup _{\substack{\psi_{h} \in \mathrm{H}_{h}^{\phi} \\
\psi_{h} \neq \mathbf{0}}} \frac{\left|A_{\boldsymbol{u}}\left(\varphi_{h}, \psi_{h}\right)-A_{\boldsymbol{u}_{h}}\left(\varphi_{h}, \psi_{h}\right)\right|}{\left\|\psi_{h}\right\|_{1, \Omega}}\right)\right\} .
\end{gathered}
$$

Next, we proceed similarly as in the proof of Lemma 3.10 to estimate each term in the foregoing equation involving a supremum. In fact, according to the definition of $G_{\phi}(c f .(3.12))$, and applying the same arguments from that proof, we readily see that

$$
\sup _{\substack{\psi_{h} \in \mathrm{H}_{h}^{\phi} \\ \psi_{h} \neq \mathbf{0}}} \frac{\left|G_{\phi}\left(\psi_{h}\right)-G_{\phi_{h}}\left(\psi_{h}\right)\right|}{\left\|\psi_{h}\right\|_{1, \Omega}} \leq L_{\gamma}\|\boldsymbol{k}\|\left\|\phi-\phi_{h}\right\|_{0, \Omega} .
$$

In turn, it is clear from the definition of $A_{\boldsymbol{u}}(c f .(3.4))$ and the estimate (3.5) that for each $\varphi_{h} \in \mathrm{H}_{h}^{\phi}$ there holds

$$
\begin{aligned}
\sup _{\substack{\psi_{h} \in \mathrm{H}_{h}^{\phi} \\
\psi_{h} \neq \mathbf{0}}} \frac{\left|A_{\boldsymbol{u}}\left(\varphi_{h}, \psi_{h}\right)-A_{\boldsymbol{u}_{h}}\left(\varphi_{h}, \psi_{h}\right)\right|}{\left\|\psi_{h}\right\|_{1, \Omega}} \leq c(\Omega)\left\|\varphi_{h}\right\|_{1, \Omega}\left\|\boldsymbol{u}-\boldsymbol{u}_{h}\right\|_{1, \Omega} \\
\leq c(\Omega)\left\|\phi-\varphi_{h}\right\|_{1, \Omega}\left\|\boldsymbol{u}-\boldsymbol{u}_{h}\right\|_{1, \Omega}+c(\Omega)\|\phi\|_{1, \Omega}\left\|\boldsymbol{u}-\boldsymbol{u}_{h}\right\|_{1, \Omega} .
\end{aligned}
$$

In this way, replacing (5.5) and (5.6) back into (5.4), we arrive at (5.3) and end the proof.

The following lemma provides a preliminary estimate for the error $\left\|(\boldsymbol{\sigma}, \boldsymbol{u})-\left(\boldsymbol{\sigma}_{h}, \boldsymbol{u}_{h}\right)\right\|_{H}$. 
Lemma 5.3. Let $C_{\mathrm{ST}}:=\alpha^{-1} \max \{1, \alpha+\|B\|\}$, where $\|B\|$ and $\alpha$ are the boundedness and ellipticity constants, respectively, of the bilinear forms $B_{\phi}$ (cf. (3.17), (3.19)). Then there holds

$$
\begin{aligned}
& \left\|(\boldsymbol{\sigma}, \boldsymbol{u})-\left(\boldsymbol{\sigma}_{h}, \boldsymbol{u}_{h}\right)\right\|_{H} \leq C_{\mathrm{ST}}\left\{(1+2\|B\|) \operatorname{dist}\left((\boldsymbol{\sigma}, \boldsymbol{u}), \mathbb{H}_{h}^{\boldsymbol{\sigma}} \times \mathbf{H}_{h}^{\boldsymbol{u}}\right)\right. \\
& \left.+\quad\left(1+\kappa_{2}^{2}\right)^{1 / 2}\|\boldsymbol{f}\|_{\infty, \Omega}\left\|\phi-\phi_{h}\right\|_{0, \Omega}+\frac{L_{\mu}\left(1+\kappa_{1}^{2}\right)^{1 / 2}}{\mu_{1}^{2}} C_{\varepsilon}\|\boldsymbol{\sigma}\|_{\varepsilon, \Omega}\left\|\phi-\phi_{h}\right\|_{\mathrm{L}^{n / \varepsilon}(\Omega)}\right\} .
\end{aligned}
$$

Proof. By applying the abstract Lemma 5.1 to the context (5.1), we obtain

$$
\begin{aligned}
& \left\|(\boldsymbol{\sigma}, \boldsymbol{u})-\left(\boldsymbol{\sigma}_{h}, \boldsymbol{u}_{h}\right)\right\|_{H} \\
& \leq C_{\mathrm{ST}}\left\{\sup _{\substack{\left(\boldsymbol{\tau}_{h}, \boldsymbol{v}_{h}\right) \in \mathbb{H}_{h} \boldsymbol{\sigma}_{h} \times \mathbf{H}_{h}^{u} \\
\left(\boldsymbol{\tau}_{h}, \boldsymbol{v}_{h}\right) \neq \mathbf{0}}} \frac{\left|F_{\phi}\left(\boldsymbol{\tau}_{h}, \boldsymbol{v}_{h}\right)-F_{\phi_{h}}\left(\boldsymbol{\tau}_{h}, \boldsymbol{v}_{h}\right)\right|}{\left\|\left(\boldsymbol{\tau}_{h}, \boldsymbol{v}_{h}\right)\right\|_{H}}\right. \\
& +\inf _{\substack{\left(\boldsymbol{\zeta}_{h}, \boldsymbol{w}_{h}\right) \in \mathbb{H}_{h} \boldsymbol{\sigma} \times \mathbf{H}_{h}^{u} \\
\left(\boldsymbol{\zeta}_{h}, \boldsymbol{w}_{h}\right) \neq \mathbf{0}}}\left(\left\|(\boldsymbol{\sigma}, \boldsymbol{u})-\left(\boldsymbol{\zeta}_{h}, \boldsymbol{w}_{h}\right)\right\|_{H}\right. \\
& \left.\left.+\sup _{\substack{\left(\boldsymbol{\tau}_{h}, \boldsymbol{v}_{h}\right) \in \mathbb{H}_{h} \boldsymbol{\sigma}_{h} \times \mathbf{H}_{h}^{u} \\
\left(\boldsymbol{\tau}_{h}, \boldsymbol{v}_{h}\right) \neq \mathbf{0}}} \frac{\left|B_{\phi}\left(\left(\boldsymbol{\zeta}_{h}, \boldsymbol{w}_{h}\right),\left(\boldsymbol{\tau}_{h}, \boldsymbol{v}_{h}\right)\right)-B_{\phi_{h}}\left(\left(\boldsymbol{\zeta}_{h}, \boldsymbol{w}_{h}\right),\left(\boldsymbol{\tau}_{h}, \boldsymbol{v}_{h}\right)\right)\right|}{\left\|\left(\boldsymbol{\tau}_{h}, \boldsymbol{v}_{h}\right)\right\|_{H}}\right)\right\} .
\end{aligned}
$$

Then, proceeding analogously as in the proof of Lemma 3.9 (cf. (3.28)), we first deduce that

$$
\sup _{\substack{\left(\boldsymbol{\tau}_{h}, \boldsymbol{v}_{h}\right) \in \mathbb{H}_{h} \times \boldsymbol{H}_{h}^{u} \\\left(\boldsymbol{\tau}_{h}, \boldsymbol{v}_{h}\right) \neq \mathbf{0}}} \frac{\left|F_{\phi}\left(\boldsymbol{\tau}_{h}, \boldsymbol{v}_{h}\right)-F_{\phi_{h}}\left(\boldsymbol{\tau}_{h}, \boldsymbol{v}_{h}\right)\right|}{\left\|\left(\boldsymbol{\tau}_{h}, \boldsymbol{v}_{h}\right)\right\|_{H}} \leq\left(1+\kappa_{2}^{2}\right)^{1 / 2}\|\boldsymbol{f}\|_{\infty, \Omega}\left\|\phi-\phi_{h}\right\|_{0, \Omega} .
$$

In turn, in order to estimate the supremum in (5.8), we add and substract suitable terms to write

$$
\begin{gathered}
B_{\phi}\left(\left(\boldsymbol{\zeta}_{h}, \boldsymbol{w}_{h}\right),\left(\boldsymbol{\tau}_{h}, \boldsymbol{v}_{h}\right)\right)-B_{\phi_{h}}\left(\left(\boldsymbol{\zeta}_{h}, \boldsymbol{w}_{h}\right),\left(\boldsymbol{\tau}_{h}, \boldsymbol{v}_{h}\right)\right)=B_{\phi}\left(\left(\boldsymbol{\zeta}_{h}, \boldsymbol{w}_{h}\right)-(\boldsymbol{\sigma}, \boldsymbol{u}),\left(\boldsymbol{\tau}_{h}, \boldsymbol{v}_{h}\right)\right) \\
+\left(B_{\phi}-B_{\phi_{h}}\right)\left((\boldsymbol{\sigma}, \boldsymbol{u}),\left(\boldsymbol{\tau}_{h}, \boldsymbol{v}_{h}\right)\right)+B_{\phi_{h}}\left((\boldsymbol{\sigma}, \boldsymbol{u})-\left(\boldsymbol{\zeta}_{h}, \boldsymbol{w}_{h}\right),\left(\boldsymbol{\tau}_{h}, \boldsymbol{v}_{h}\right)\right),
\end{gathered}
$$

whence, applying the boundedness (3.17) to the first and third terms on the right hand side of the foregoing equation, and proceeding analogously as for the derivation of (3.29) and (3.30) with the second one, we find that

$$
\begin{aligned}
& \sup _{\substack{\left(\boldsymbol{s}_{h}, \boldsymbol{v}_{h}\right) \in \mathbb{H} \boldsymbol{H}_{h} \times \mathbf{H}_{h}^{u} \\
\left(\boldsymbol{\tau}_{h}, \boldsymbol{v}_{h}\right) \neq \mathbf{0}}} \frac{\left|B_{\phi}\left(\left(\boldsymbol{\zeta}_{h}, \boldsymbol{w}_{h}\right),\left(\boldsymbol{\tau}_{h}, \boldsymbol{v}_{h}\right)\right)-B_{\phi_{h}}\left(\left(\boldsymbol{\zeta}_{h}, \boldsymbol{w}_{h}\right),\left(\boldsymbol{\tau}_{h}, \boldsymbol{v}_{h}\right)\right)\right|}{\left\|\left(\boldsymbol{\tau}_{h}, \boldsymbol{v}_{h}\right)\right\|_{H}} \\
& \quad \leq 2\|B\|\left\|(\boldsymbol{\sigma}, \boldsymbol{u})-\left(\boldsymbol{\zeta}_{h}, \boldsymbol{w}_{h}\right)\right\|_{H}+\frac{L_{\mu}\left(1+\kappa_{1}^{2}\right)^{1 / 2}}{\mu_{1}^{2}} C_{\varepsilon}\|\boldsymbol{\sigma}\|_{\varepsilon, \Omega}\left\|\phi-\phi_{h}\right\|_{L^{n / \varepsilon}(\Omega)} .
\end{aligned}
$$

Finally, by replacing (5.9) and (5.10) into (5.8), we arrive at (5.7), which ends the proof.

We now combine the inequalities provided by Lemmas 5.2 and 5.3 to derive the Céa estimate for the total error $\left\|\phi-\phi_{h}\right\|_{1, \Omega}+\left\|(\boldsymbol{\sigma}, \boldsymbol{u})-\left(\boldsymbol{\sigma}_{h}, \boldsymbol{u}_{h}\right)\right\|_{H}$. To this end, and in order to simplify the subsequent writing, we introduce the following constants

$$
C_{1}:=\widetilde{C}_{\mathrm{ST}} L_{\gamma}, \quad C_{2}:=\widetilde{C}_{\mathrm{ST}} c(\Omega) r C_{\mathrm{ST}}\left(1+\kappa_{2}^{2}\right)^{1 / 2}, \quad C_{3}:=\widetilde{C}_{\mathrm{ST}} c(\Omega) r C_{\mathrm{ST}} \frac{L_{\mu}\left(1+\kappa_{1}^{2}\right)^{1 / 2}}{\mu_{1}^{2}} C_{\varepsilon} \widetilde{C}_{S}(r) \widetilde{C}_{\varepsilon} .
$$


Hence, by replacing the bound for $\left\|\boldsymbol{u}-\boldsymbol{u}_{h}\right\|_{1, \Omega}$ given by (5.7) into the second term on the right hand side of (5.3), recalling that $\|\phi\|_{1, \Omega} \leq r$, employing from (3.22) that

$$
\|\boldsymbol{\sigma}\|_{\varepsilon, \Omega} \leq \widetilde{C}_{S}(r)\left\{\left\|\boldsymbol{u}_{D}\right\|_{1 / 2+\varepsilon, \Gamma_{\mathrm{D}}}+\|\boldsymbol{f}\|_{\infty, \Omega}\|\phi\|_{0, \Omega}\right\},
$$

using again that $\widetilde{C}_{\varepsilon}$ is the boundedness constant of the continuous injection of $\mathrm{H}^{1}(\Omega)$ into $\mathrm{L}^{n / \varepsilon}(\Omega)$, and performing several algebraic manipulations, we can assert that

$$
\begin{aligned}
\| \phi & -\phi_{h}\left\|_{1, \Omega} \leq\left\{C_{1}\|\boldsymbol{k}\|+\left(C_{2}+r C_{3}\right)\|\boldsymbol{f}\|_{\infty, \Omega}+C_{3}\left\|\boldsymbol{u}_{\mathrm{D}}\right\|_{1 / 2+\varepsilon, \Gamma_{\mathrm{D}}}\right\}\right\| \phi-\phi_{h} \|_{1, \Omega} \\
& +\widetilde{C}_{\mathrm{ST}} c(\Omega) r C_{\mathrm{ST}}(1+2\|B\|) \operatorname{dist}\left((\boldsymbol{\sigma}, \boldsymbol{u}), \mathbb{H}_{h}^{\boldsymbol{\sigma}} \times \mathbf{H}_{h}^{\boldsymbol{u}}\right) \\
& +\widetilde{C}_{\mathrm{ST}}\left(1+c(\Omega)\left\|\boldsymbol{u}-\boldsymbol{u}_{h}\right\|_{1, \Omega}\right) \operatorname{dist}\left(\phi, \mathrm{H}_{h}^{\phi}\right) .
\end{aligned}
$$

Note here that $\|\boldsymbol{u}\|_{1, \Omega}$ and $\left\|\boldsymbol{u}_{h}\right\|_{1, \Omega}$ are estimated according to (3.16), and hence the expression in (5.11) multiplying $\operatorname{dist}\left(\phi, \mathrm{H}_{h}^{\phi}\right)$ is already controlled by constants, parameters, and data only. As a consequence of the foregoing discussion, we can establish the following result providing the requested Céa estimate.

Theorem 5.4. Assume that the data $\boldsymbol{k}, \boldsymbol{f}$, and $\boldsymbol{u}_{\mathrm{D}}$ are sufficiently small so that

$$
C_{1}\|\boldsymbol{k}\|+\left(C_{2}+r C_{3}\right)\|\boldsymbol{f}\|_{\infty, \Omega}+C_{3}\left\|\boldsymbol{u}_{\mathrm{D}}\right\|_{1 / 2+\varepsilon, \Gamma_{\mathrm{D}}}<\frac{1}{2} .
$$

Then, there exist positive constants $C_{4}$ and $C_{5}$, depending only on parameters, data, and other constants, all them independent of $h$, such that

$$
\left\|\phi-\phi_{h}\right\|_{1, \Omega}+\left\|(\boldsymbol{\sigma}, \boldsymbol{u})-\left(\boldsymbol{\sigma}_{h}, \boldsymbol{u}_{h}\right)\right\|_{H} \leq C_{4} \operatorname{dist}\left((\boldsymbol{\sigma}, \boldsymbol{u}), \mathbb{H}_{h}^{\boldsymbol{\sigma}} \times \mathbf{H}_{h}^{\boldsymbol{u}}\right)+C_{5} \operatorname{dist}\left(\phi, \mathrm{H}_{h}^{\phi}\right) .
$$

Proof. The estimate for $\left\|\phi-\phi_{h}\right\|_{1, \Omega}$ follows straightforwardly from (5.11) and (5.12), and then, the replacement of it back into (5.7), using also that $\left\|\phi-\phi_{h}\right\|_{L^{n / \varepsilon}(\Omega)} \leq \widetilde{C}_{\varepsilon}\left\|\phi-\phi_{h}\right\|_{1, \Omega}$, completes the proof.

We end this section with the corresponding rates of convergence of our Galerkin scheme (4.4).

Theorem 5.5. In addition to the hypotheses of Theorems 3.13, 4.7, and 5.4, assume that there exists $s>0$ such that $\boldsymbol{\sigma} \in \mathbb{H}^{s}(\Omega), \operatorname{div} \boldsymbol{\sigma} \in \mathbf{H}^{s}(\Omega), \boldsymbol{u} \in \mathbf{H}^{1+s}(\Omega)$, and $\phi \in \mathrm{H}^{1+s}(\Omega)$. Then, there exists $\widehat{C}>0$, independent of $h$, such that, with the finite element subspaces defined by (4.1), (4.2), and (4.3), there holds

$$
\begin{aligned}
& \left\|\phi-\phi_{h}\right\|_{1, \Omega}+\left\|(\boldsymbol{\sigma}, \boldsymbol{u})-\left(\boldsymbol{\sigma}_{h}, \boldsymbol{u}_{h}\right)\right\|_{H} \\
& \quad \leq \widehat{C} h^{\min \{s, k+1\}}\left\{\|\boldsymbol{\sigma}\|_{s, \Omega}+\|\operatorname{div} \boldsymbol{\sigma}\|_{s, \Omega}+\|\boldsymbol{u}\|_{1+s, \Omega}+\|\phi\|_{1+s, \Omega}\right\} .
\end{aligned}
$$

Proof. It follows directly from the Céa estimate (5.13) and the approximation properties of $\mathbb{H}_{h}^{\sigma}, \mathbf{H}_{h}^{u}$, and $\mathrm{H}_{h}^{\phi}$ (cf. $[5,10,21])$.

\section{NumERICAL RESUlts}

We illustrate the performance of our mixed-primal finite element method with some numerical tests. We first study the accuracy of the approximations by manufacturing an exact solution of the nonlinear problem (2.1) defined on $\Omega=(0,1)^{2}$. We introduce the coefficients $\mu(\phi)=(1-c \phi)^{-2}, \gamma(\phi)=c \phi(1-c \phi)^{2}, \vartheta(|\nabla \phi|)=$ 

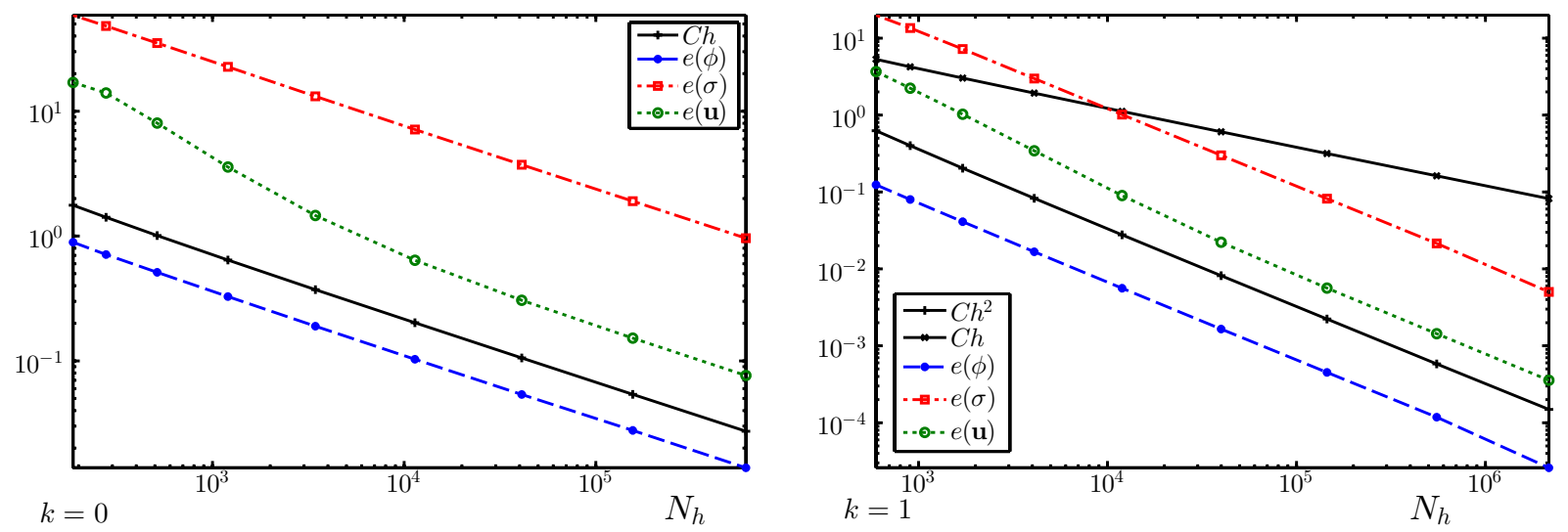

Figure 1. Example 1: computed errors $e(\phi), e(\boldsymbol{\sigma}), e(\boldsymbol{u})$ associated to the mixed-primal approximation versus the number of degrees of freedom $N_{h}$ for $\mathrm{RT}_{0}-\mathbf{P}_{1}-\mathrm{P}_{1}$ and $\mathrm{RT}_{1}-\mathbf{P}_{2}-\mathrm{P}_{2}$ finite elements (left and right, respectively). See values in Table 1. (In color online).

$m_{1}+m_{2}\left(1+|\nabla \phi|^{2}\right)^{m_{3} / 2-1}$, and the source terms on the right hand sides are adjusted in such a way that the exact solutions are given by the smooth functions

$$
\begin{aligned}
& \phi\left(x_{1}, x_{2}\right)=b-b \exp \left(-x_{1}\left(x_{1}-1\right) x_{2}\left(x_{2}-1\right)\right), \quad \boldsymbol{u}\left(x_{1}, x_{2}\right)=\left(\begin{array}{c}
\sin \left(2 \pi x_{1}\right) \cos \left(2 \pi x_{2}\right) \\
-\cos \left(2 \pi x_{1}\right) \sin \left(2 \pi x_{2}\right)
\end{array}\right), \\
& \boldsymbol{\sigma}\left(x_{1}, x_{2}\right)=2 \pi\left(\begin{array}{l}
\frac{\cos \left(2 \pi x_{1}\right) \cos \left(2 \pi x_{2}\right)}{\left(1-b c+b c e^{-x_{1}\left(x_{1}-1\right) x_{2}\left(x_{2}-1\right)}\right)^{2}} \frac{-\sin \left(2 \pi x_{1}\right) \sin \left(2 \pi x_{2}\right)}{\left(1-b c+b c e^{-x_{1}\left(x_{1}-1\right) x_{2}\left(x_{2}-1\right)}\right)^{2}} \\
\frac{\sin \left(2 \pi x_{1}\right) \sin \left(2 \pi x_{2}\right)}{\left(1-b c+b c e^{-x_{1}\left(x_{1}-1\right) x_{2}\left(x_{2}-1\right)}\right)^{2}} \frac{-\cos \left(2 \pi x_{1}\right) \cos \left(2 \pi x_{2}\right)}{\left(1-b c+b c e^{-x_{1}\left(x_{1}-1\right) x_{2}\left(x_{2}-1\right)}\right)^{2}}
\end{array}\right)-\left(x_{1}^{2}-x_{2}^{2}\right) \mathbb{I},
\end{aligned}
$$

for $\left(x_{1}, x_{2}\right) \in \bar{\Omega}$. We take $b=15, c=m_{1}=m_{2}=1 / 2, m_{3}=3 / 2$ and set $\Gamma_{\mathrm{D}}=\partial \Omega$, where $\phi$ vanishes and $\boldsymbol{u}_{D}$ is imposed accordingly to the exact solution. The mean value of $\operatorname{tr} \boldsymbol{\sigma}_{h}$ over $\Omega$ is fixed via a penalization strategy. As defined above, the scalar field $\phi$ is bounded in $\Omega$ and so the coefficients are also bounded. In particular we have $\mu_{1}=0.99$ and $\mu_{2}=3.35$. Therefore, and as suggested by Lemma 3.4, the stabilization constants are chosen as $\kappa_{1}=\mu_{1}^{2} / \mu_{2}=0.2976, \kappa_{2}=1 / \mu_{2}=0.2985$, and $\kappa_{3}=\kappa_{1} / 2=0.1488$.

The domain is partitioned into quasi-uniform meshes with $2^{n}+3, n=0,1, \ldots, 8$ vertices on each side of the domain. The convergence of the approximate solutions is assessed by computing errors in the respective norms and experimental rates, that we define as usual

$$
\begin{array}{ll}
e(\boldsymbol{\sigma}):=\left\|\boldsymbol{\sigma}-\boldsymbol{\sigma}_{h}\right\|_{\operatorname{div}, \Omega}, \quad e(\boldsymbol{u}):=\left\|\boldsymbol{u}-\boldsymbol{u}_{h}\right\|_{1, \Omega}, \quad e(\phi):=\left\|\phi-\phi_{h}\right\|_{1, \Omega}, \\
r(\boldsymbol{\sigma}):=\frac{\log (e(\boldsymbol{\sigma}) / \hat{e}(\boldsymbol{\sigma}))}{\log (h / \hat{h})}, \quad r(\boldsymbol{u}):=\frac{\log (e(\boldsymbol{u}) / \hat{e}(\boldsymbol{u}))}{\log (h / \hat{h})}, \quad r(\phi):=\frac{\log (e(\phi) / \hat{e}(\phi))}{\log (h / \hat{h})},
\end{array}
$$

where $e$ and $\hat{e}$ denote errors computed on two consecutive meshes of sizes $h$ and $\hat{h}$, respectively. Notice that these errors are computed between the finite element approximation and the corresponding interpolate of the exact solution. Values and plots of errors and corresponding rates associated to $\mathrm{RT}_{k}-\mathbf{P}_{k+1}-\mathrm{P}_{k+1}$ approximations with $k=0$ and $k=1$ are summarized in Table 1 and Figure 1, respectively, where we observe convergence rates of $O\left(h^{k+1}\right)$ for stresses, velocities and the scalar field in the relevant norms. These findings are in agreement with the theoretical error bounds of Section 5 ( $c f .(5.14)$ ). A Newton-Raphson's algorithm with a tolerance of $1 \mathrm{E}-08$ has been applied to the resolution of the nonlinear problem (4.4), and at each iteration the linear systems resulting from the linearization were solved by means of the multifrontal massively parallel solver (MUMPS [3]). 
TABLE 1. Example 1: convergence history and Newton iteration count for the mixed-primal $\mathrm{RT}_{k}-\mathbf{P}_{k+1}-\mathrm{P}_{k+1}$ approximations of the coupled problem, $k=0,1$. Here $N_{h}$ stands for the number of degrees of freedom associated to each triangulation $\mathcal{T}_{h}$.

\begin{tabular}{|c|c|c|c|c|c|c|c|c|}
\hline$N_{h}$ & $h$ & $e(\phi)$ & $r(\phi)$ & $e(\boldsymbol{\sigma})$ & $r(\sigma)$ & $e(\boldsymbol{u})$ & $r(\boldsymbol{u})$ & iter \\
\hline \multicolumn{9}{|c|}{ Augmented $\mathrm{RT}_{0}-\mathbf{P}_{1}-\mathrm{P}_{1}$ scheme } \\
\hline 187 & 0.353553 & 0.891473 & - & 58.80212 & - & 16.97841 & - & 8 \\
\hline 278 & 0.282843 & 0.711188 & 0.970463 & 48.21425 & 0.852938 & 13.99512 & 1.014962 & 7 \\
\hline 514 & 0.202031 & 0.512189 & 0.975540 & 35.19082 & 0.935794 & 8.041585 & 1.424675 & 7 \\
\hline 1202 & 0.128565 & 0.327347 & 0.990462 & 22.67913 & 0.972039 & 3.573343 & 1.579459 & 7 \\
\hline 3442 & 0.074432 & 0.189813 & 0.997142 & 13.16677 & 0.994888 & 1.461483 & 1.563582 & 6 \\
\hline 11378 & 0.040406 & 0.103089 & 0.999241 & 7.138732 & 1.002043 & 0.639297 & 1.235346 & 6 \\
\hline 41074 & 0.021107 & 0.053859 & 0.999801 & 3.722753 & 1.002661 & 0.305779 & 1.113577 & 6 \\
\hline 155762 & 0.010795 & 0.027705 & 0.999948 & 1.904552 & 1.002240 & 0.152283 & 1.034021 & 6 \\
\hline 606322 & 0.005460 & 0.013933 & 0.999987 & 0.961174 & 1.001041 & 0.076408 & 1.010863 & 6 \\
\hline \multicolumn{9}{|c|}{ Augmented $\mathrm{RT}_{1}-\mathbf{P}_{2}-\mathrm{P}_{2}$ scheme } \\
\hline 595 & 0.353553 & 0.123752 & - & 19.88141 & - & 3.675443 & - & 7 \\
\hline 903 & 0.282843 & 0.079988 & 1.955574 & 13.55213 & 1.717465 & 2.237812 & 2.223581 & 6 \\
\hline 1711 & 0.202031 & 0.041028 & 1.984189 & 7.213065 & 1.874291 & 1.026756 & 2.215637 & 6 \\
\hline 4095 & 0.128565 & 0.016689 & 1.990120 & 2.989083 & 1.949025 & 0.343355 & 2.223416 & 6 \\
\hline 11935 & 0.074432 & 0.005607 & 1.995567 & 1.012340 & 1.981522 & 0.089977 & 2.150313 & 6 \\
\hline 39903 & 0.040406 & 0.001654 & 1.998442 & 0.299392 & 1.994287 & 0.022247 & 2.187332 & 6 \\
\hline 144991 & 0.021107 & 0.000451 & 1.999545 & 0.081778 & 1.998531 & 0.005629 & 2.116371 & 6 \\
\hline 551775 & 0.010795 & 0.000118 & 1.999836 & 0.021401 & 1.999468 & 0.001439 & 2.034801 & 6 \\
\hline 2164783 & 0.005460 & 0.000026 & 1.999935 & 0.005014 & 2.006076 & 0.000357 & 2.013878 & 6 \\
\hline
\end{tabular}

We mention that an average number of 7 Newton steps were required to reach the desired tolerance. All remaining examples were carried out using $k=0$, i.e., lowest-order Raviart-Thomas finite element approximations for the rows of the Cauchy stress tensor, and piecewise linear approximations of velocity components and the scalar field $\phi$. The augmented mixed-primal approximations computed on a mesh of 37249 vertices and 74496 elements are depicted in Figure 2.

In our second example we assess the capability of a 3D implementation by carrying out the benchmark test of thermal convection on the cube $\Omega=(0,1)^{3}$ (see e.g. $[19,26]$ ). The relevant equations, here written in terms of stresses $\boldsymbol{\sigma}$, velocities $\boldsymbol{u}$, and temperature $\phi$ correspond to the Boussinesq approximation and can be readily recovered from (2.5) by setting $g=0, \boldsymbol{f} \phi=\frac{1}{\rho}(0, \phi-1,0)^{\mathrm{t}}, \mu(\phi)=\operatorname{Re}^{-1}=(\operatorname{Ra} / \operatorname{Pr})^{-1 / 2}, \vartheta(\phi)=(\operatorname{Re} \operatorname{Pr})^{-1}$, $\gamma(\phi)=0$, where $\operatorname{Pr}=0.71, \operatorname{Ra}=1 \mathrm{E} 05$, and $\rho=0.1$ are the Prandtl (ratio between the viscous and thermal diffusions), Rayleigh (only parameter remaining after nondimensionalization of the Boussinesq approximation), and overheat ratio coefficients, respectively. Notice that this problem is linear, except for the convection term. Even if the problem setting does not coincide exactly with the case analyzed previously, our goal is to illustrate the applicability of the present coupling strategy in diverse scenarios. In fact, if we redefine $\boldsymbol{f}:=\frac{1}{\rho}(0,1,0)^{\mathrm{t}}$, then the functional (3.9) will eventually contain two additional terms independent of $\boldsymbol{f}$, and all the subsequent continuous and discrete analysis would remain unchanged after replacing $\boldsymbol{f} \phi$ by $\boldsymbol{f} \phi-\boldsymbol{f}$.

The stabilization constants are chosen as $\kappa_{1}=\mu, \kappa_{2}=1 / \mu$, and $\kappa_{3}=\mu / 2$. As boundary data we impose $\boldsymbol{u}_{D}=\mathbf{0}$ on the whole $\partial \Omega$, whereas we put $\phi=(2-\rho) / 2$ at $x_{1}=0$ and $\phi=(2+\rho) / 2$ at $x_{1}=1$. On the remainder of $\partial \Omega$ we impose zero-flux conditions for $\phi$, that is $\tilde{\boldsymbol{\sigma}} \cdot \boldsymbol{\nu}=0$. The domain is discretized on a mesh $\mathcal{T}_{h}$ of 46656 vertices and 271950 tetrahedra, and we represent the field quantities of interest in Figure 3. From these plots we can observe a satisfactory qualitative agreement with respect to published data (see e.g. $[14,19,26]$ ). 


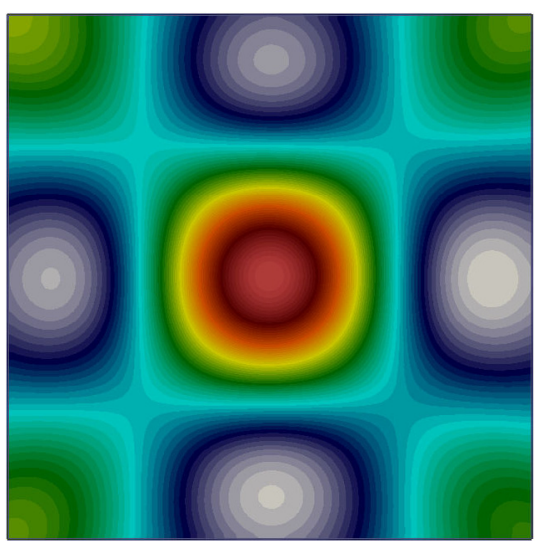

0

$\sigma_{11, h}$

$-7.97$

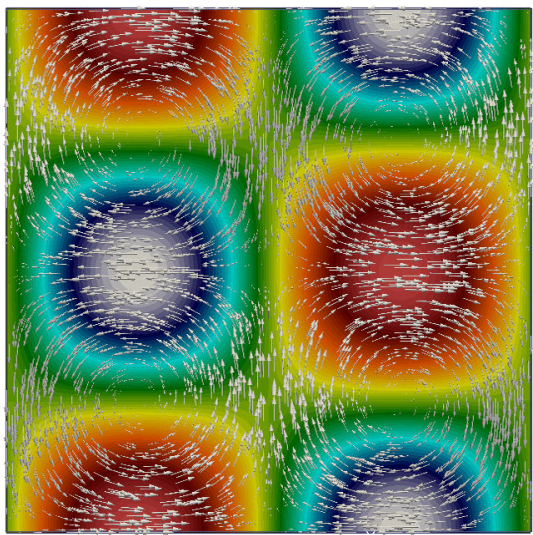

$u_{1, h}$

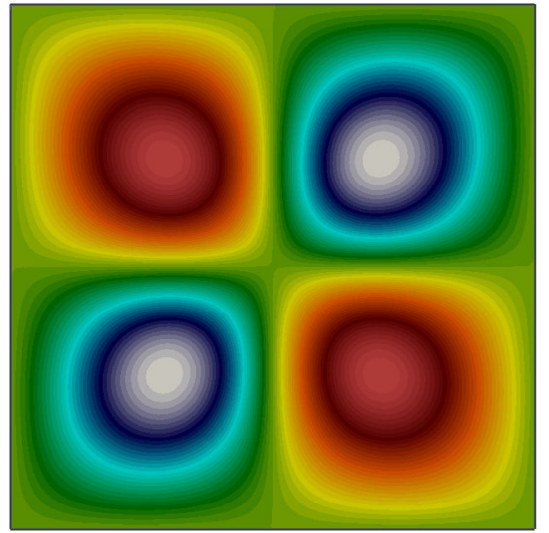

0

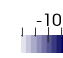

$-12.4$

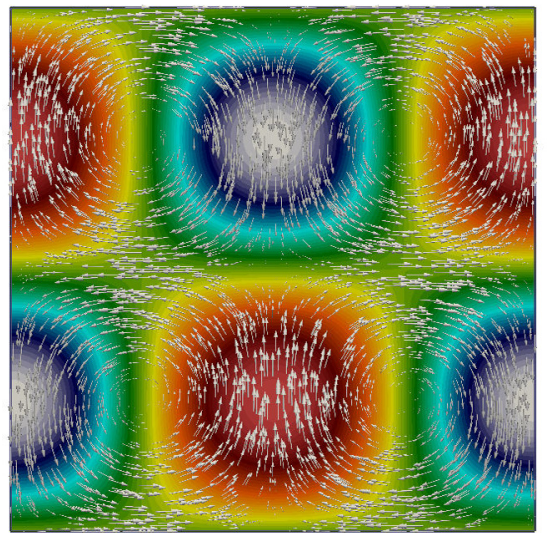

$u_{2, h}$

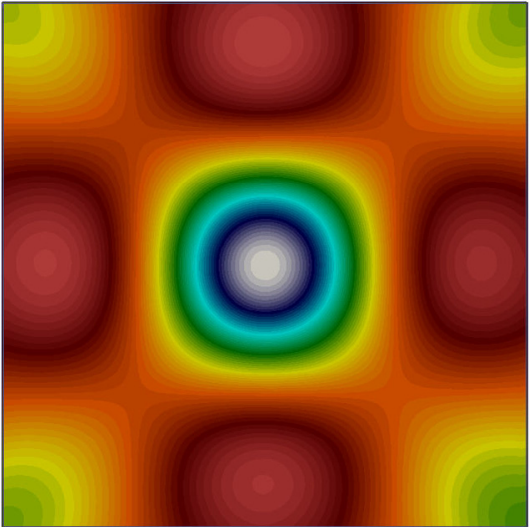

$-10$

0
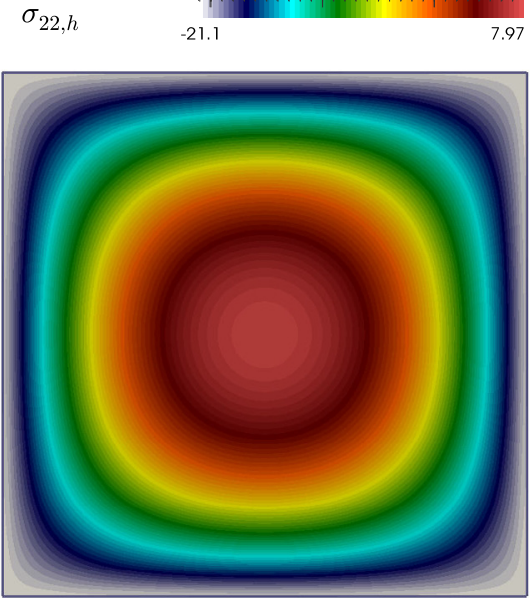

$\phi_{h}$

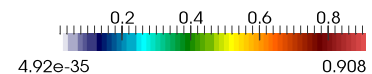

Figure 2. Example 1: $\mathrm{RT}_{0}-\mathbf{P}_{1}-\mathrm{P}_{1}$ approximation of stress components $\boldsymbol{\sigma}_{h}$ (top panels), velocity components $\boldsymbol{u}_{h}$ (with vector directions, bottom left and center, respectively), and scalar field $\phi_{h}$ (bottom right) solving (3.10). The mesh has 37249 vertices and 74496 triangular elements. (In color online).

Moreover, Figure 4 reports on the mid-plane $\left(x_{3}=0.5\right)$ profiles and a comparison with respect to values described in [19], including the average Nusselt number associated to a plane $S$ (at fixed $x_{1}$ ) and computed as $\mathrm{Nu}=\int_{S} \operatorname{Pr} \operatorname{Re} u_{1} \phi-\partial_{1} \phi$. Our findings, after an average of 9 Newton iterations to reach a tolerance of $1 \mathrm{E}-08$, satisfactorily match the benchmark data in terms of maximum and minimum velocities and temperature profiles at the symmetry lines $x_{1}=0.5$ and $x_{2}=0.5$. More quantitative comparisons are also presented in Table 2 , where we have collected some outputs of interest for different values of the Rayleigh number. For larger Rayleigh numbers, an homotopy (or continuation) method was carried out on the Rayleigh number in order to ensure convergence of the algorithm.

Our last example focuses on the simulation of the steady state of a clarifying-thickening process. The basin, the different boundaries of the geometry, and the generated volumetric mesh consisting of 64135 vertices and 370597 tetrahedra are sketched in Figure 5. The size of the mesh and the finite element choice (row-wise lowestorder Raviart-Thomas's approximations for stresses and piecewise linear elements for velocity components and 

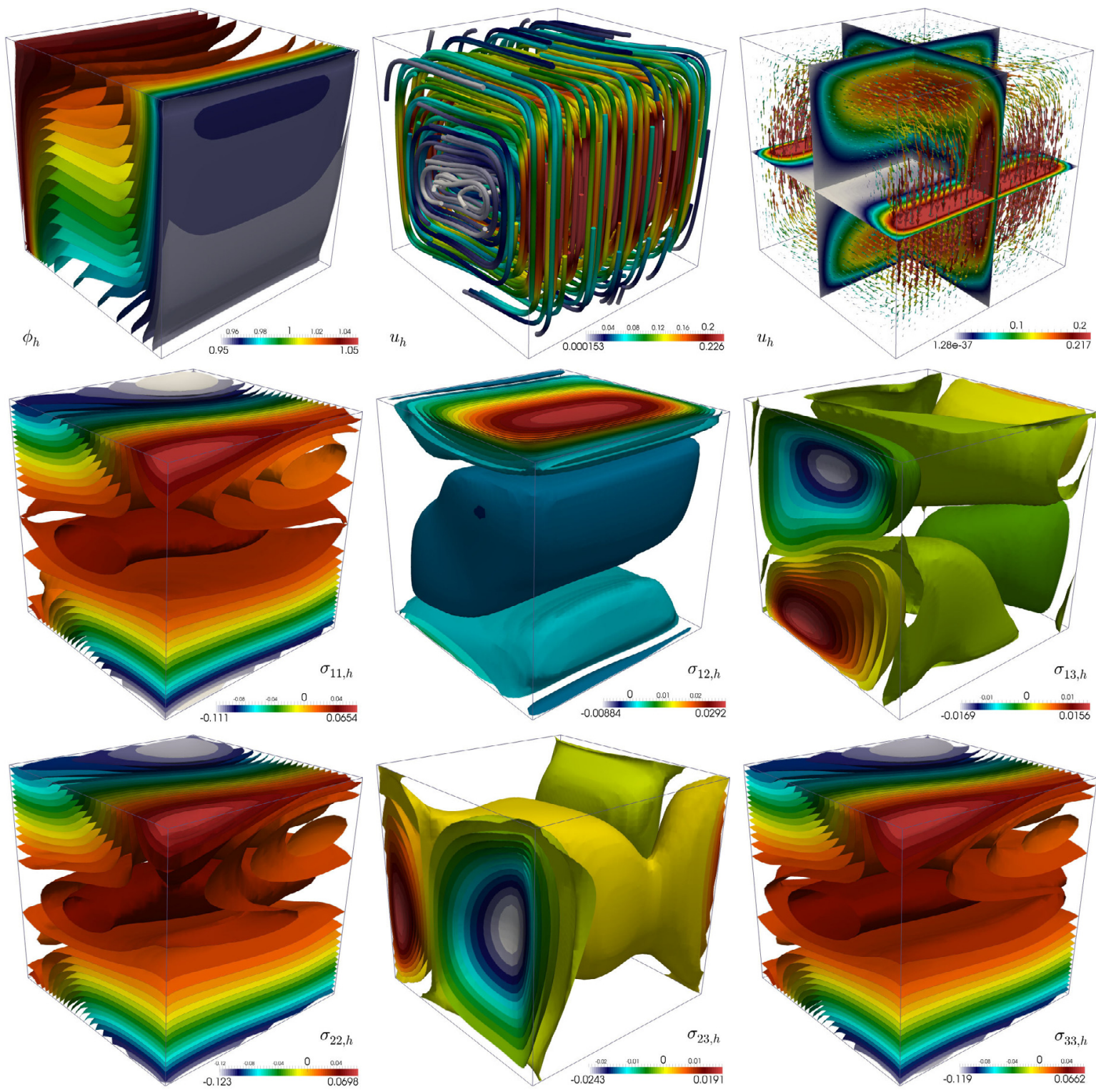

Figure 3. Example 2: computed temperature iso-surfaces (top left) and velocity streamlines and vectors colored by magnitude (top center and right, respectively) and principal components of the Cauchy stress (center and bottom rows) for the thermal cavity test. (In color online).

concentration) implies that at each Newton step we solve for a total of 2515211 degrees of freedom. The nonlinear functions of the concentration are taken as in [7]: $\mu(\phi)=\left(1-\phi / \phi_{\max }\right)^{-2.5}, \gamma(\phi)=u_{\infty}\left(1+\phi\left(1-\phi / \phi_{\max }\right)\right)^{2}$, $\vartheta(\phi)=\frac{\gamma(\phi) \sigma_{0} \alpha\left(\phi / \phi_{c}\right)^{\alpha-1}}{\phi \phi_{c} G \Delta \rho}+u_{\infty}$ and the source terms are $\boldsymbol{f}=(0,0,-G)^{\mathrm{t}}, g=0$. The physical values assumed by the concentration (it remains bounded between 0 and $\phi_{\max }$ ) imply that the viscosity, hindered flux, and compressibility coefficients satisfy $(2.2)$ and (2.3) with $\mu_{1}=1, \mu_{2}=2.7, \gamma_{1}=u_{\infty}, \gamma_{2}=1.15 u_{\infty}, \vartheta_{1}=4.28, \vartheta_{2}=$ 29.74. However, notice that $\vartheta$ depends explicitly on $\phi$ and not on the concentration gradient, which was not 

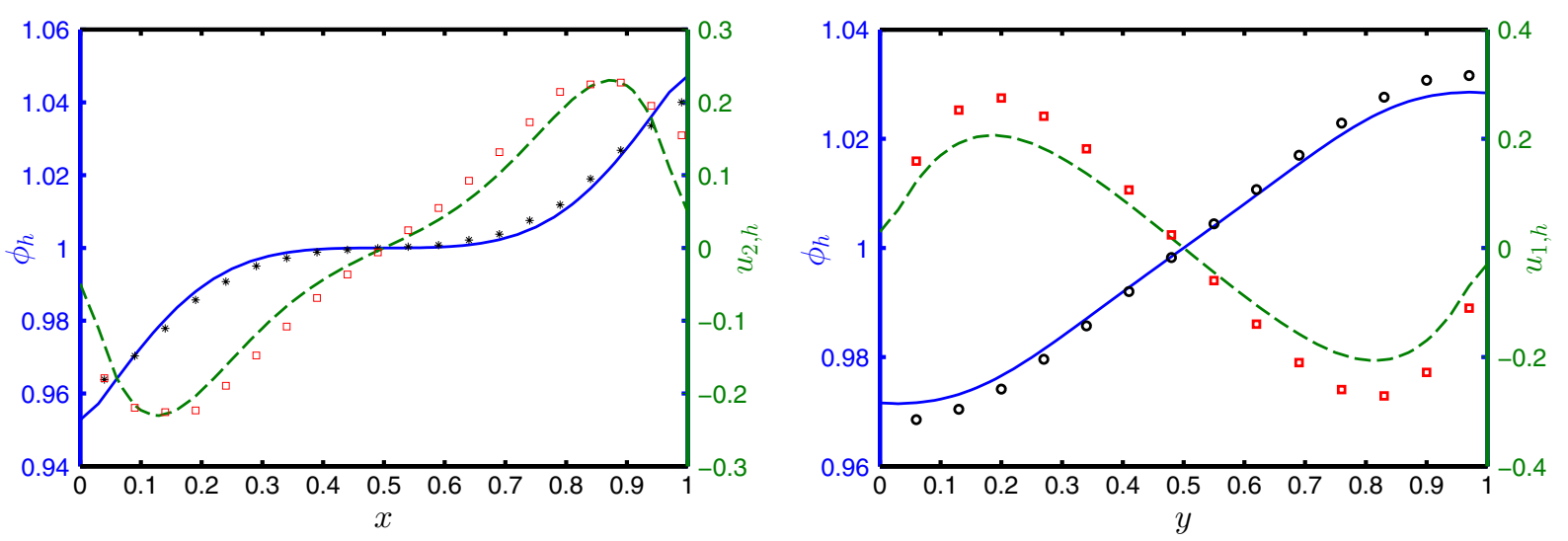

Figure 4. Example 2: temperature profiles (solid blue, left axis) and velocity components (dashed green, right axis) at $x_{3}=0.5$, and comparison with respect to benchmark solutions (In color online).

TABle 2. Example 2: outputs of interest (Nusselt number, maximum value of the normalized horizontal velocity on the mid-plane attained at $\left(0.5, x_{2}^{\infty}, 0.5\right)$, and maximum value of the normalized vertical velocity and its position $\left(x_{1}^{\infty}, 0.5,0.5\right)$ on the central horizontal plane, respectively) for different values of the Rayleigh number, and comparison with respect to values from $[14,19]$.

\begin{tabular}{lcccccc}
\hline & $\mathrm{Ra}$ & $\mathrm{Nu}$ & $\max \left(\left|\hat{u}_{1, h}\right|\right)$ & $\max \left(\left|\hat{u}_{2, h}\right|\right)$ & $x_{1}^{\infty}$ & $x_{2}^{\infty}$ \\
\hline Computed & $10^{3}$ & 1.134 & 0.129 & 0.131 & 0.176 & 0.845 \\
{$[14]$} & $10^{3}$ & 1.117 & 0.136 & 0.138 & 0.178 & 0.813 \\
{$[19]$} & $10^{3}$ & - & 0.132 & 0.131 & 0.200 & 0.833 \\
Computed & $10^{4}$ & 2.030 & 0.195 & 0.229 & 0.121 & 0.819 \\
{$[14]$} & $10^{4}$ & 2.054 & 0.192 & 0.234 & 0.119 & 0.823 \\
{$[19]$} & $10^{4}$ & 2.100 & 0.201 & 0.225 & 0.117 & 0.817 \\
Computed & $10^{5}$ & 4.321 & 0.145 & 0.244 & 0.064 & 0.843 \\
{$[14]$} & $10^{5}$ & 4.337 & 0.153 & 0.261 & 0.066 & 0.855 \\
{$[19]$} & $10^{5}$ & 4.361 & 0.147 & 0.247 & 0.065 & 0.855 \\
\hline
\end{tabular}
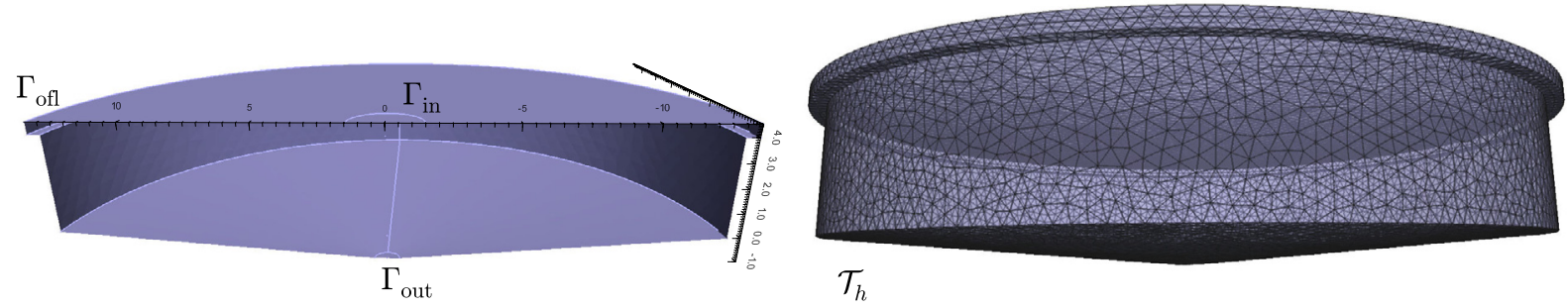

FIGURE 5. Example 3: geometry of the clarifier-thickener unit (left panel) and tetrahedral mesh $\mathcal{T}_{h}$ with 64135 vertices and 370597 elements (right panel). (In color online). 

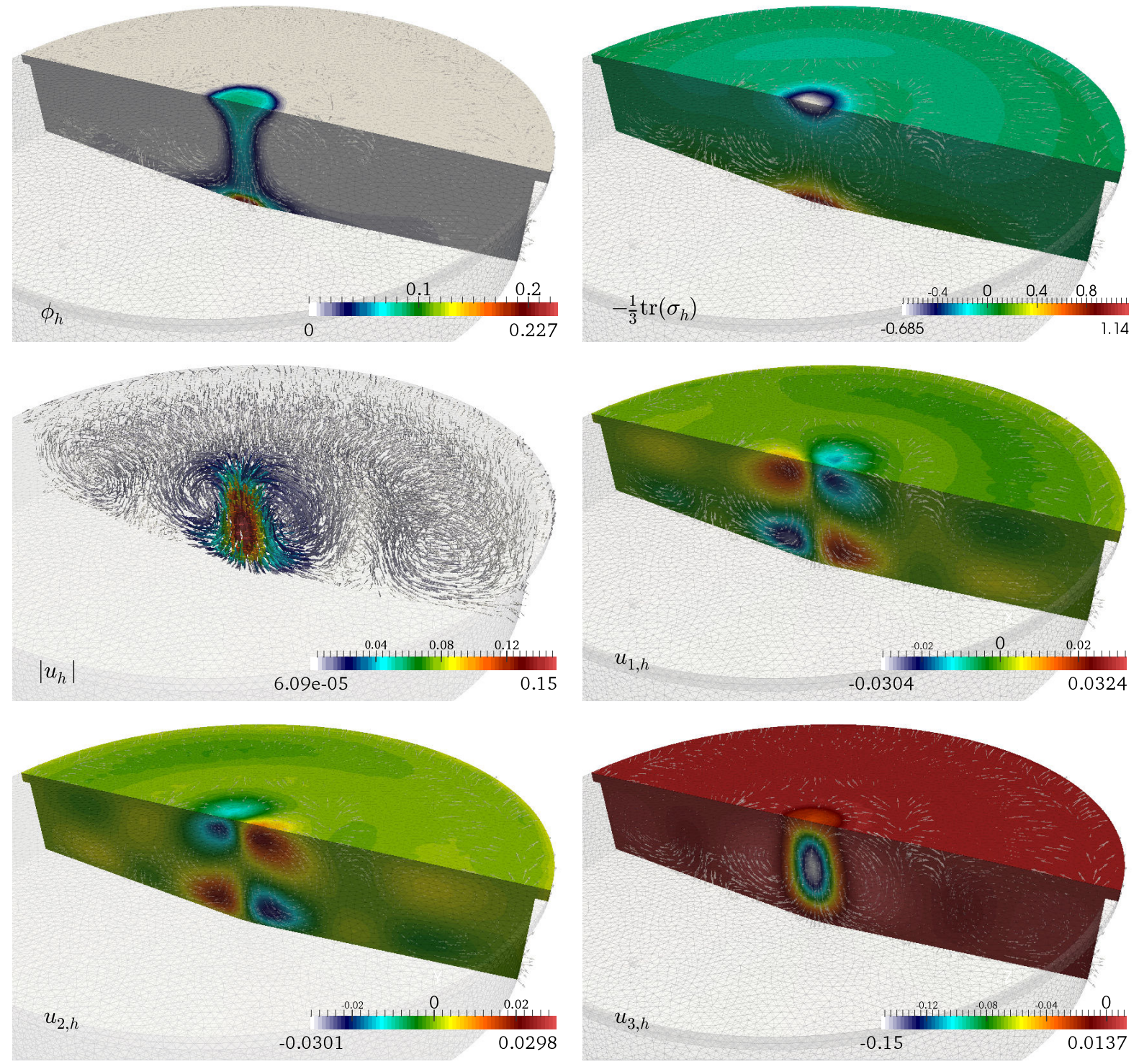

Figure 6. Example 3: simulation of a clarifier-thickener unit. From left-top: approximated concentration profile, opposite of the trace of the Cauchy stress tensor (which corresponds to the suggested approximation of the pressure field), and velocity components (color online).

addressed in the solvability analysis of the model problem. While one could try to analyze this case by using some classical results on pseudomonotone operators (see, e.g. [11], Sect. 9.3), ([27], Sect. 3.3)), in the forthcoming work [2] we have chosen to extend the present approach to this modified model since in this way we are able to derive not only the existence of continuous and discrete solutions but also the corresponding a priori error analysis.

Boundary conditions are set as follows: concentration and velocities are fixed on the inlet disc $\Gamma_{\text {in }}$ according to $\phi=\phi_{\text {in }}$ and $\boldsymbol{u}=\boldsymbol{u}_{\text {in }}=\left(0,0,-u_{3, \text { in }}\right)^{\mathrm{t}}$. At the outlet disk $\Gamma_{\text {out }}$ we prescribe $\boldsymbol{u}=\boldsymbol{u}_{\text {out }}=\left(0,0,-u_{3, \text { out }}\right)^{\mathrm{t}}$, at the overflow annulus we do not constraint the velocity field, and on the remainder of $\partial \Omega$ we put no slip boundary 
data for the velocity and zero-flux conditions for the concentration. Model parameters are set as $u_{3, \text { in }}=1.29 \mathrm{E}-02$, $u_{3, \text { out }}=2.54 \mathrm{E}-03, \Delta \rho=1562, \phi_{\max }=0.9, \phi_{c}=0.1, u_{\infty}=2.2 \mathrm{E}-03, G=9.81, \phi_{\text {in }}=0.08, \alpha=5$, and $\sigma_{0}=5 \mathrm{E}-02$. We mimic the behavior of a transient simulation by adding a mass term $\eta \phi$ to the concentration equation, with $\eta=1 \mathrm{E}-03$. Such a modification does not entail a major change in the analysis: it suffices to replace the part of the flux $\phi \boldsymbol{u}$ by $\phi(\boldsymbol{u}+\eta)$.

According to the bounds of the viscosity, the stabilization parameters were set as $\kappa_{1}=\kappa_{2}=0.4784$, and $\kappa_{3}=0.2392$. We mention that 8 Newton iterations were needed to achieve a tolerance of $1 \mathrm{E}-07$ for the energy norm of the incremental approximations. The numerical results are depicted in Figure 6 (we show half of the tank for visualization purposes), including concentration profile, velocity vectors, pressure approximation (computed in terms of the trace of the Cauchy stress), and velocity components. We can observe that the material is removed from the unit at the boundary $\Gamma_{\text {out }}$ with concentration $\phi \approx 0.24$, which agrees with the results in e.g. ([6], Example 3).

Acknowledgements. The authors are very thankful to both referees for helpful indications and remarks, and particularly to one of them who suggested key arguments to improve the analysis and results of our paper.

\section{REFERENCES}

[1] R.A. Adams and J.J.F. Fournier, Sobolev Spaces. Academic Press. Elsevier Ltd (2003).

[2] M. Alvarez, G.N. Gatica and R. Ruiz-Baier, Mixed-primal finite element approximation of a steady sedimentation-consolidation system. In preparation (2015).

[3] P.R. Amestoy, I.S. Duff and J.-Y. L'Excellent, Multifrontal parallel distributed symmetric and unsymmetric solvers. Comput. Methods Appl. Mech. Engrg. 184 (2000) 501-520.

[4] C. Amrouche, C. Bernardi, M. Dauge and V. Girault, Vector potentials in three-dimensional non-smooth domains. Math. Methods Appl. Sci. 21 (1998) 823-864.

[5] F. Brezzi and M. Fortin, Mixed and Hybrid Finite Element Methods. Springer-Verlag (1991).

[6] R. Bürger, R. Ruiz-Baier and H. Torres, A stabilized finite volume element formulation for sedimentation-consolidation processes. SIAM J. Sci. Comput. 34 (2012) B265-B289.

[7] R. Bürger, W.L. Wendland and F. Concha, Model equations for gravitational sedimentation-consolidation processes. ZAMM Z. Angew. Math. Mech. 80 (2000) 79-92.

[8] M.C. Bustos, F. Concha, R. Bürger and E.M. Tory, Sedimentation and Thickening. Kluwer Academic Publishers, Dordrecht (1999).

[9] Z. Cai, B. Lee and P. Wang, Least-squares methods for incompressible Newtonian fluid flow: linear stationary problems. SIAM J. Numer. Anal. 42 (2004) 843-859.

[10] P. Ciarlet, The Finite Element Method for Elliptic Problems. North-Holland (1978).

[11] P. Ciarlet, Linear and Nonlinear Functional Analysis with Applications. Society for Industrial and Applied Mathematics, Philadelphia, PA (2013).

[12] E. Colmenares, G.N. Gatica and R. Oyarzúa, Analysis of an augmented mixed-primal formulation for the stationary Boussinesq problem. Centro de Investigación en Ingeniería Matemática, Universidad de Concepción, (2014). Preprint 2015-07. Available at http://www.ci2ma.udec.cl/publicaciones/prepublicaciones/.

[13] C. Cox, H. Lee and D. Szurley, Finite element approximation of the non-isothermal Stokes-Oldroyd equations. Int. J. Numer. Anal. Model. 4 (2007) 425-440.

[14] G. de Vahl Davis, Natural convection of air in a square cavity: A benchmark numerical solution. Int. J. Numer. Meth. Fluids 3 (1983) 249-264.

[15] M. Farhloul and A. Zine, A dual mixed formulation for non-isothermal Oldroyd-Stokes problem. Math. Model. Nat. Phenom. 6 (2011) $130-156$.

[16] M. Farhloul, S. Nicaise and L. Paquet, A mixed formulation of Boussinesq equations: Analysis of nonsingular solutions. Math. Comput. 69 (2000) 965-986.

[17] L.E. Figueroa, G.N. Gatica and N. Heuer, A priori and a posteriori error analysis of an augmented mixed finite element method for incompressible fluid flows. Comput. Methods Appl. Mech. Engrg. 198 (2008) 280-291.

[18] L.E. Figueroa, G.N. Gatica and A. Márquez, Augmented mixed finite element methods for the stationary Stokes equations. SIAM J. Sci. Comput. 31 (2008/09) 1082-1119.

[19] T. Fusegi and J.M. Hyun, A numerical study of 3D natural convection in a cube: effects of the horizontal thermal boundary conditions. Fluid Dyn. Res. 8 (1991) 221-230.

[20] G.N. Gatica, Analysis of a new augmented mixed finite element method for linear elasticity allowing $\mathbb{R}_{0}-\mathbb{P}_{1}-\mathbb{P}_{0}$ approximations. ESAIM: M2AN 40 (2006) 1-28.

[21] G.N. Gatica, A Simple Introduction to the Mixed Finite Element Method: Theory and Applications. Springer Briefs in Mathematics. Springer, Cham (2014). 
[22] G.N. Gatica and G.C. Hsiao, On the coupled BEM and FEM for a nonlinear exterior Dirichlet problem in R². Numer. Math. 61 (1992) 171-214.

[23] G.N. Gatica and W. Wendland, Coupling of mixed finite elements and boundary elements for linear and nonlinear elliptic problems. Appl. Anal. 63 (1996) 39-75.

[24] G.N. Gatica, A. Márquez and M.A. Sánchez, Analysis of a velocity-pressure-pseudostress formulation for the stationary Stokes equations. Comput. Methods Appl. Mech. Engrg. 199 (2010) 1064-1079.

[25] G.N. Gatica, R. Oyarzúa and F.-J. Sayas, A twofold saddle point approach for the coupling of fluid flow with nonlinear porous media flow. IMA J. Numer. Anal. 32 (2012) 845-887.

[26] Y. Le Pentrec and G. Lauriat, Effects of the heat transfer at the side walls on natural convection in cavities. J. Heat Trans. 112 (1990) 370-378.

[27] J. Nečas, Introduction to the Theory of Nonlinear Elliptic Equations. Reprint of the 1983 edition. A Wiley-Interscience Publication. John Wiley \& Sons, Ltd., Chichester (1986).

[28] R. Oyarzúa, T. Qin and D. Schötzau, An exactly divergence-free finite element method for a generalized Boussinesq problem. IMA J. Numer. Anal. 34 (2014) 1104-1135.

[29] A. Quarteroni and A. Valli, Numerical Approximation of Partial Differential Equations. Vol. 23 of Springer Ser. Comput. Math. Springer-Verlag Berlin Heidelberg (1994).

[30] R. Ruiz-Baier and H. Torres, Numerical solution of a multidimensional sedimentation problem using finite volume-element methods. Appl. Numer. Math. 95 (2015) 280-291.

[31] S.B. Savage, Gravity flow of cohesionless granular materials in chutes and channels. J. Fluid Mech. 92 (1979) 53-96. 